Review

\title{
Modeling of granular solids with computational homogenization: Comparison with Biot's theory
}

\author{
F. Marinelli ${ }^{\text {a,*, }}$, A.P. van den Eijnden ${ }^{\text {b }}$, Y. Sieffert ${ }^{c, d}$, R. Chambon ${ }^{c, d}$, F. Collin ${ }^{e}$ \\ ${ }^{a}$ Northwestern University, Department of Civil and Environmental Engineering, 2145 Sheridan Road Tech A124, IL 60208-3109 Evanston, USA \\ ${ }^{\mathrm{b}}$ Faculty of Civil Engineering and Geoscience, Delft University of Technology, Stevinweg 1, 2628 CN Delft, The Netherlands \\ ${ }^{\text {c } U n i v e r s i t e ́ ~ G r e n o b l e ~ A l p e s, ~ L a b o r a t o i r e ~ 3 S-R ~ F-38000 ~ G r e n o b l e, ~ F r a n c e ~}$ \\ ${ }^{\mathrm{d}}$ CNRS, 3SR F-38000 Grenoble, France \\ e Université de Liège, Département ArGEnCo/Géotechnique, Institut de Mécanique et Génie civil, Chemin des Chevreuils 1, 4000 Liege 1, Belgique
}

\section{A R T I C L E I N F O}

\section{Article history:}

Received 30 November 2015

Received in revised form

23 April 2016

Accepted 16 May 2016

\section{Keywords:}

Biot's theory

Computational homogenization $\left(\mathrm{FE}^{2}\right)$

Hydromechanical modeling

Cohesive materials

Poromechanics

Periodic medium

Microstructure

\begin{abstract}
A B S T R A C T
This paper discusses the numerical results for a consolidation test studied by using a hydromechanical model formulated within a numerical homogenization approach, the so-called finite element squared method, $\mathrm{FE}^{2}$. This model is characterized by two observation scales: at the microscopic scale, the microstructure of the material is described as an assembly of hyperelastic grains connected by cohesive interfaces that define a network of channels in which fluid can percolate. This microstructure, periodically distributed in the small-scale, identifies the Representative Elementary Volume of the material. At the macroscopic scale, the material is treated as a continuum and the corresponding constitutive equations are obtained by means of a numerical homogenization process on the microscopic problem. In this manner, the total stress of the mixture, the density of the mixture, the fluid mass flow and the fluid mass content can be computed. The objective of this work is to compare the numerical results with the analytical solution of a classical oedometric test using the poroelastic theory of Biot (1941) [1]. For this purpose, it is shown that the hydromechanical behavior obtained with the selected $\mathrm{FE}^{2}$ method is characterized by a classical Biot-like porous medium and the resulting macroscopic properties can be illustrated in light of the hydromechanical mechanisms at the microscopic scale.
\end{abstract}

\section{Contents}

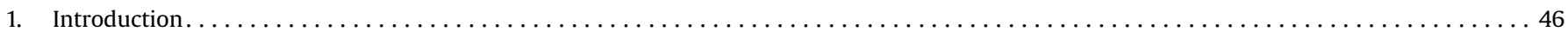

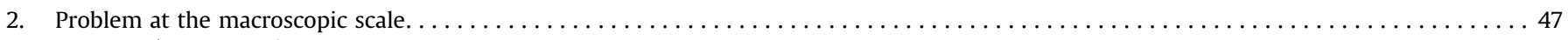

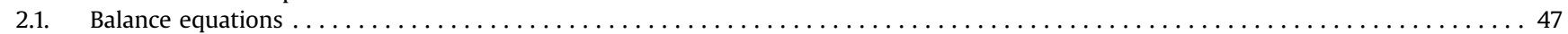

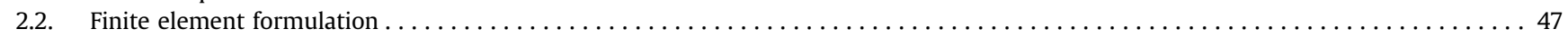

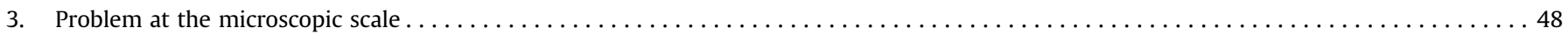

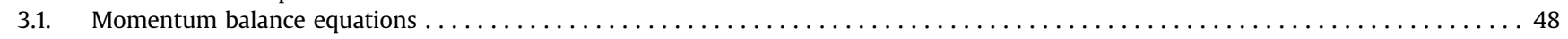

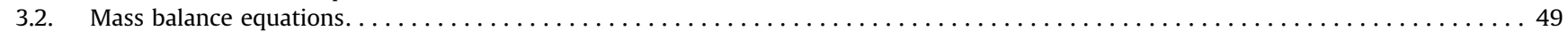

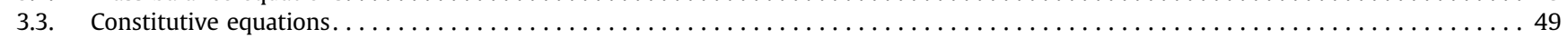

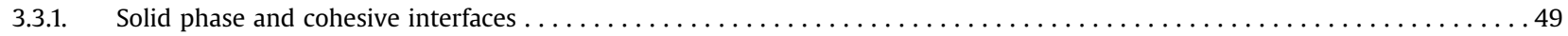

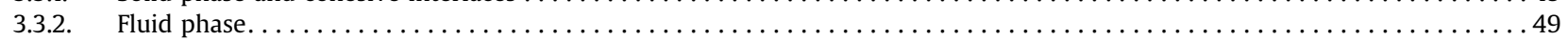

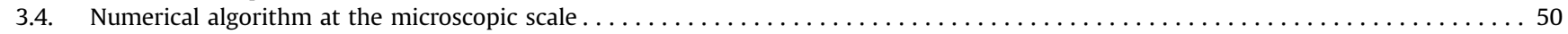

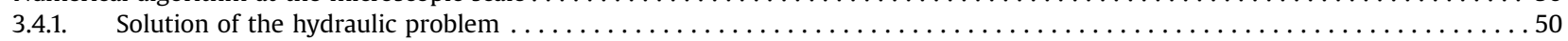

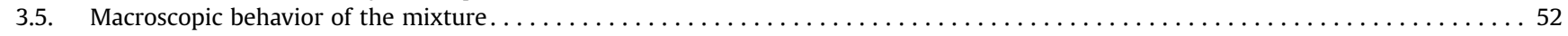

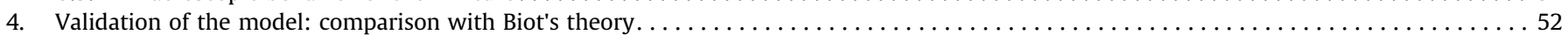

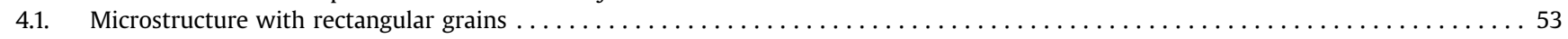

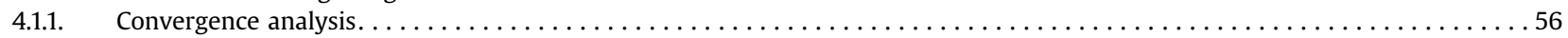

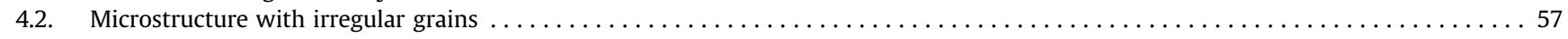

\footnotetext{
* Corresponding author.

E-mail addresses: ferdinando.marinelli@northwestern.edu (F. Marinelli), a.p.vandeneijnden@tudelft.nl (A.P. van den Eijnden), yannick.sieffert@3sr-grenoble.fr(Y. Sieffert), rene.chambon@3sr-grenoble.fr (R. Chambon), f.collin@ulg.ac.be (F. Collin).
} 


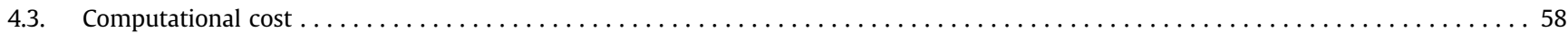

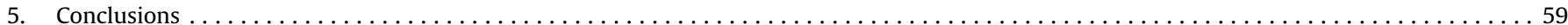

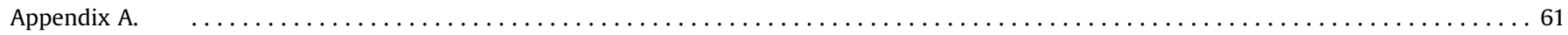

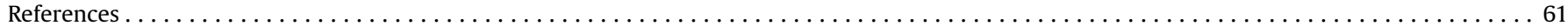

\section{Introduction}

The investigation of the material behavior at small observation scales represents one of the modern goals of geomechanics to interpret macroscopic mechanisms of granular and cohesive geomaterials. In the last decades, advanced experimental techniques have been developed to capture the local deformation field of laboratory samples and to explore complex microscopic fabrics of soils and rock-like materials [2,3]. Such advanced tools can provide useful information to model material heterogeneities at small observation scales and, consequently, to derive macroscopic constitutive relations by means of upscaling techniques.

In the past, several analytical methods have been considered to upscale the microscopic stress field for random and periodic heterogeneous media. In this framework, it is worth mentioning the method based on averaging theory [4-6] and the self-consistent method [7-11]. In the former approach, the overall properties of the equivalent continuum are obtained by performing an average operation on the microconstituents of the material. In the latter, the macroscopic properties are computed by using analytical solutions of a continuum with spherical and ellipsoidal inclusions in an infinite matrix with different properties. A different analytical upscaling technique is the asymptotic expansions theory, firstly developed by Auriault and Sanchez-Palencia [12-14]. In this approach, the Representative Elementary Volume is identified by a periodic unit-cell mimicking the microstructure of the material (Fig. 1) for which the homogenized macroscopic properties are derived by using a variational formulation on a local microscopic boundary value problem. A complete review detailing several homogenization methods can be found in [15].

The mentioned methods are very efficient for linear problems in which the microstructure is schematized with simple geometries. For complex microstructures and non-linear behavior, the analytical approach is difficult to apply even though some authors have obtained interesting results [16]. Consistently with the hypothesis of periodic microstructure and scale separability, a different procedure is the one in which the upscaling is performed with numerical computations, the so-called finite element squared $\mathrm{FE}^{2}$ method. In this method, also called global-local analysis [17], the material point behavior is defined via a numerical homogenization process by performing a finite element computation upon a given microstructure which identifies the Representative
Elementary Volume of the material. Originally proposed by Terada and Kikuchi [17] to model the non-linear behavior of composite materials, the $\mathrm{FE}^{2}$ method was extended to complex microscopic constitutive equations in which finite plasticity [18,19] and viscoplasticity [20-22] have been taken into account. To include in the model size-effects and highly microstructural heterogeneities, second order terms were introduced by Kouznetsova et al. [23,24] in which a length scale was derived from micromechanical computations by adding the gradient of the macroscopic deformation tensor to the boundary conditions of the REV (see also [25] and [26]). A comprehensive review about the computational homogenization method for monophasic materials can be found in Schröder [27].

In the framework of multiphysical coupling, the $\mathrm{FE}^{2}$ method was at first used by Özdemir et al. [28] to model thermomechanical effects of heat conduction problems. Other authors have extended this approach to deal with problems in which the solid structure is saturated with a fluid phase. In this context, the hydromechanical behavior of fully saturated porous media has been studied by Su et al. [29] and by Massart and Selvadurai [30]. In particular, in this last work, the effective porosity and the permeability evolution are derived with numerical computations on a given microstructure.

In the presented study, the microscopic model proposed by Frey et al. [31], which was used only at the material point level, is here implemented to solve an initial boundary value problem where the hydromechanical constitutive equations are defined with double-scale computations ( $\mathrm{FE}^{2}$ method). In particular, to demonstrate the consistency of the selected approach, a consolidation problem is studied and the obtained results are compared with the corresponding one-dimensional analytical solution within the classical poroelastic theory of Biot [1]. For this purpose, the computed equivalent porous medium is characterized with the homogenized hydromechanical properties obtained through numerical computations. This comparison shows that the macroscopic response, based on an explicit modeling of the microstructure, is described by a classical Biot-like porous medium and can be considered as a further validation of the implemented microscopic model in the finite element code Lagamine (code of University of Liège, Belgium, [32,33]). Similar motivations were considered by Catalano et al. [34] to validate and to interpret a
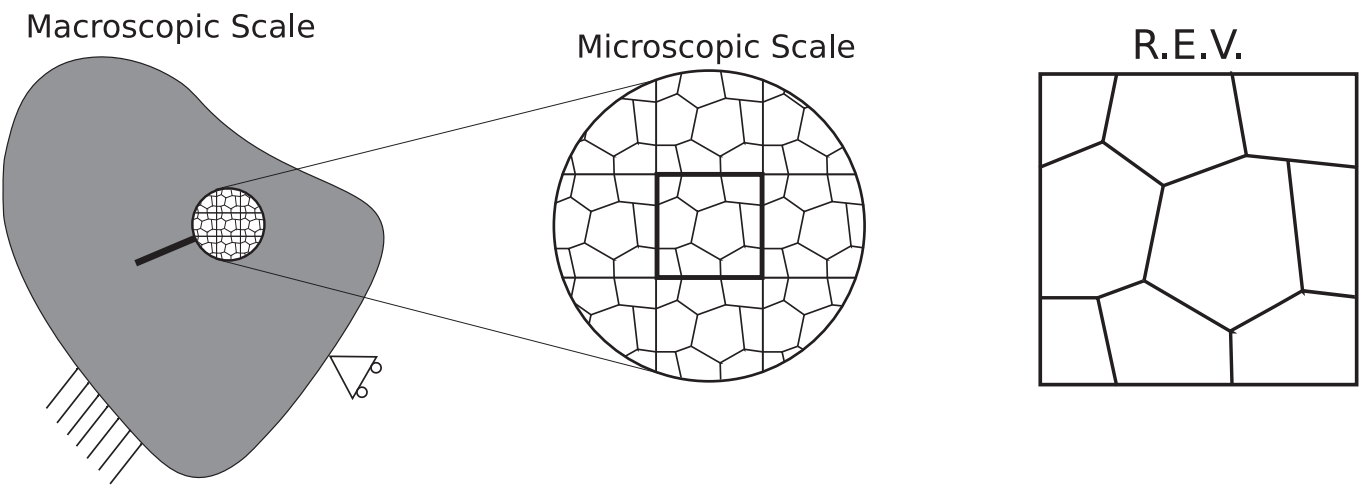

Fig. 1. Observation scales for finely periodic continuum. 
micro-hydromechanical model implemented with the discrete element method.

This paper is organized as follows: the main notions of a coupled finite element problem used at the macroscale are recalled in Section 2 to highlight some key differences with classical phenomenological approaches and to introduce some quantities computed during the homogenization process. In Section 3, the microscopic model is presented to point out some changes with respect to the work of Frey et al. [31] and to detail the numerical algorithm of the small-scale problem which is not extensively presented in [31]. The numerical results of a double-scale problem are discussed in the fourth section where, for validation purposes, the macroscopic constitutive relations are derived for two different microstructures.

Henceforth, vectors and tensors are denoted with lower case indices and the classical summation convention with respect to repeated tensorial indices is used.

\section{Problem at the macroscopic scale}

To introduce the hydromechanical problem solved at the macroscopic scale, the equations solving an initial boundary value problem by using the finite element method [35] are recalled in this section. Consistently with classical poromechanics theory [3640], a mixture of a solid skeleton saturated by one fluid is considered. The momentum balance equation and the mass conservation equation, both written in the current solid configuration denoted $\Omega^{t}$ (updated Lagrangian configuration), have to be enforced for the solid and the fluid phases. The balance of momentum for the fluid phase is written in a strong form in which volume forces and the gradient of water pressure are equilibrated by drag forces. The momentum balance equation for the skeleton and the mass conservation equation for the fluid are written in a weak form with the assumption of quasi-static conditions.

The field equations are then linearized and solved numerically within a full Newton-Raphson algorithm. The linearization is performed directly in the continuous problem by deriving the socalled linear auxiliary system [41], similarly to the method proposed by Borja and Alarcón [42] and Borja et al. [43] in the framework of finite elastoplasticity. Hereafter, to adapt the macroscopic finite element algorithm for double-scale computations and to emphasize some key differences with classical phenomenological approaches, the linear auxiliary system is expressed in a general form without introducing a particular set of constitutive assumptions to describe the behavior of the porous medium.

\subsection{Balance equations}

The balance equation of momentum for the mixture and the mass balance equation for the fluid, both written in the weak form, are recalled hereafter. In this paper, the superscript $t$ means "at time $t$ ". For any virtual displacement field $u_{i}^{\star}$, the momentum balance equation of the mixture can be written as follows:

$\int_{\Omega^{t}} \sigma_{i j}^{t} \frac{\partial u_{i}^{\star}}{\partial x_{j}^{t}} d \Omega^{t}=\int_{\Omega^{t}} e^{m i x, t} g_{i} u_{i}^{\star} d \Omega^{t}+\int_{\Gamma_{\sigma}^{t}} \bar{t}_{i} u_{i}^{\star} d \Gamma^{t}$,

where $\sigma_{i j}{ }^{t}$ means the Cauchy stress of the mixture, $\varrho^{\text {mix, } t}$ is the mass density of the mixture, $\Omega^{t}$ is the current volume of a given mass of the solid skeleton and $\Gamma_{\sigma}^{t}$ is the part of the boundary where classical traction forces $\bar{t}_{i}^{t}$ are known. $g_{i}$ represents the components of the gravity. For any admissible fluid pore pressure field $p^{\star}$, the mass balance equation of the fluid is written in Eq. (2):

$\int_{\Omega^{t}}\left(\dot{M}^{t} p^{\star}-m_{i}^{t} \frac{\partial p^{\star}}{\partial x_{i}^{t}}\right) d \Omega^{t}=\int_{\Omega^{t}} Q^{t} p^{\star} d \Omega^{t}-\int_{\Gamma_{q}^{t}} \bar{q}^{t} p^{\star} d \Gamma^{t}$, $m_{i}$ is the mass flow of the fluid, $M^{t}$ is the fluid mass inside the current configuration of the skeleton $\Omega^{t}$. A dot denotes the time derivative. $Q^{t}$ is a sink term and $\Gamma_{q}^{t}$ is the part of the boundary where an input fluid mass per unit area $\bar{q}^{t}$ is prescribed.

\subsection{Finite element formulation}

To solve the non-linear system of Eqs. (1) and (2), a NewtonRaphson algorithm is employed. For this purpose, time is discretized in a sequence of steps $\Delta t$ and, assuming that a configuration $\Omega^{\tau 1}$ close to the solution is known, it is possible to define the residuals $R^{\tau 1}$ and $W^{\tau 1}$ as follows:

$\int_{\Omega^{\tau 1}} \sigma_{i j}^{\tau 1} \frac{\partial u_{i}^{\star}}{\partial x_{j}^{\tau 1}} d \Omega^{\tau 1}-\int_{\Omega^{\tau 1}} \varrho^{m i x, \tau 1} g_{i} u_{i}^{\star} d \Omega^{\tau 1}-\int_{\Gamma_{\sigma}^{\tau 1}} \bar{t}_{i} u_{i}^{\star} d \Gamma^{\tau 1}=R^{\tau 1}$,

$\int_{\Omega^{\tau 1}}\left(\dot{M}^{\tau 1} p^{\star}-m_{i}^{\tau 1} \frac{\partial p^{\star}}{\partial x_{i}^{\tau 1}}\right) d \Omega^{\tau 1}-\int_{\Omega^{\tau 1}} Q^{\tau 1} p^{\star} d \Omega^{\tau 1}-\int_{\Gamma_{q}^{\tau 1}} \bar{q}^{\tau 1} p^{\star} d \Gamma^{\tau 1}=W^{\tau 1}$

In order to compute a well balanced configuration $\Omega^{\tau 2}$ for which the residuals $R^{\tau 2}$ and $W^{\tau 2}$ vanish, the field equations (3) and (4) are linearized following the mathematical strategy detailed in [44] and [41]. As a result, a consistent linearization for the constitutive equations of the mixture lead to define a linear auxiliary system of the Newton-Raphson method as:

$\int_{\Omega^{\tau 1}}\left[U_{(x, y)}^{\star, \tau 1}\right]_{(1 \times 9)}^{\top}\left[E_{(x, y)}^{\tau 1}\right]_{(9 \times 9)}\left[\mathrm{d} U_{(x, y)}^{\tau 1}\right]_{(9 \times 1)} d \Omega^{\tau 1}=-R^{\tau 1}-W^{\tau 1}$,

where $\left[\mathrm{d} U_{(x, y)}^{\tau 1}\right]$ is defined in Eq. (6) and the vector $\left[U_{(x, y)}^{\star, \tau 1}\right]$ represents the kinematically admissible virtual variations of $\left[\mathrm{d} U_{(x, y)}^{\tau 1}\right]$.

$\left[\mathrm{d} U_{(x, y)}^{\tau 1}\right]_{i}=\left[\frac{\partial \mathrm{d} u_{1}^{\tau 1}}{\partial x_{1}^{\tau 1}} \frac{\partial \mathrm{d} u_{1}^{\tau 1}}{\partial x_{2}^{\tau 1}} \frac{\partial \mathrm{d} u_{2}^{\tau 1}}{\partial x_{1}^{\tau 1}} \frac{\partial \mathrm{d} u_{2}^{\tau 1}}{\partial x_{2}^{\tau 1}} \mathrm{~d} u_{1}^{\tau 1} \mathrm{~d} u_{2}^{\tau 1} \frac{\partial \mathrm{d} p^{\tau 1}}{\partial x_{1}^{\tau 1}} \frac{\partial \mathrm{d} p^{\tau 1}}{\partial x_{2}^{\tau 1}} \mathrm{~d} p^{\tau 1}\right]$,

The matrix $\left[E_{(x, y)}^{\tau 1}\right]$ represents the local stiffness matrix of the problem and can be expressed as follows:

$\left[E^{\tau 1}\right]=\left[\begin{array}{ccccccccc}C_{1111} & C_{1112} & C_{1121} & C_{1122} & 0 & 0 & B_{111} & B_{112} & A_{11} \\ C_{1211} & C_{1212} & C_{1221} & C_{1222} & 0 & 0 & B_{121} & B_{122} & A_{12} \\ C_{2111} & C_{2112} & C_{2121} & C_{2122} & 0 & 0 & B_{211} & B_{212} & A_{21} \\ C_{2211} & C_{2212} & C_{2221} & C_{2222} & 0 & 0 & B_{221} & B_{222} & A_{22} \\ D_{11} g_{1} & D_{12} g_{1} & D_{21} g_{1} & D_{22} g_{1} & 0 & 0 & G_{1} g_{1} & G_{2} g_{1} & E g_{1} \\ D_{11} g_{2} & D_{12} g_{2} & D_{21} g_{2} & D_{22} g_{2} & 0 & 0 & G_{1} g_{2} & G_{2} g_{2} & E g_{2} \\ K_{111} & K_{112} & K_{121} & K_{122} & 0 & 0 & N_{11} & N_{12} & L_{1} \\ K_{211} & K_{212} & K_{221} & K_{222} & 0 & 0 & N_{21} & N_{22} & L_{2} \\ H_{11} & H_{12} & H_{21} & H_{22} & 0 & 0 & J_{1} & J_{2} & F\end{array}\right]$

The matrix $N_{i j}$, the vectors $L_{i}$ and $J_{i}$ and the scalar quantity $F$ define the stiffness matrix for a fluid problem. The matrices $B_{i j k}, A_{i j}$, $K_{i j k}$ and $H_{i j}$ represent the stiffness matrices containing all coupling terms between the mechanical and the hydraulic part of the numerical problem. $C_{i j k l}$ gives the mechanical behavior of the skeleton.

As can be observed in Eq. (7), the local stiffness matrix is reported by including all the possible sources of coupling between the mechanical and the hydraulic responses. In the classical poromechanics theory, these coupling terms are developed analytically as a consequence of the phenomenological hypothesis describing the constitutive behavior of the skeleton, the solid and the fluid phase, and their reciprocal interactions [41,45]. In the framework of the $\mathrm{FE}^{2}$, the constitutive assumptions are here addressed exclusively to describe the microscopic behavior of the material. As a result, the coupling terms appearing in the matrix 
$E_{i j}^{\tau 1}$ represent an outcome of the numerical upscaling process performed for a given microstructure. For this reason, the components of the local stiffness matrix cannot be analytically developed and have to be implemented in its general form, as reported in Eq. (7). The classical perturbation method will be used to compute the local stiffness matrix $E^{\tau 1}$ and the results are presented in Section 4.

To solve an initial boundary value problem with the finite element method, the domain is discretized in finite elements and the element stiffness matrix is computed by introducing the transformation matrices $\left[T^{\tau 1}\right]$ and $[B]$ (i.e., $\mathrm{d} U_{(x, y)}^{\tau 1}=\left[T^{\tau 1}\right] \mathrm{d} U_{(\xi, \eta)}^{\tau 1}$ and $\left.\mathrm{d} U_{(\xi, \eta)}^{\tau 1}=[B] \mathrm{d} U_{n d}^{\tau 1}\right)$ in Eq. (5):

$$
\begin{aligned}
{\left[U_{n d}^{\star}\right]^{\top} } & \left\{\int_{-1}^{+1} \int_{-1}^{+1}\left([B]^{\top}\left[T^{\tau 1}\right)^{\top}\left[E_{(x, y)}^{\tau 1}\right]\left[T^{\tau 1}\right][B]\right] \operatorname{det}(J)^{\tau 1} \mathrm{~d} \xi \mathrm{d} \eta\right\}\left[\mathrm{d} U_{n d}^{\tau 1}\right] \\
& \equiv\left[U_{n d}^{\star}\right]^{\top}\left[k_{e}^{\tau 1}\right]\left[\mathrm{d} U_{n d}^{\tau 1}\right],
\end{aligned}
$$

where $\left[k_{e}^{\tau 1}\right]$ is the element stiffness matrix, $J^{\tau 1}$ is the Jacobian matrix of the mapping from $(\xi, \eta)$ to $(x, y)$ and $\left[\mathrm{d} U_{n d}^{\tau 1}\right]$ is the vector of all degrees of freedom defined on the parent element. The residuals $R^{\tau 1}$ and $W^{\tau 1}$ are computed as follows:

$$
\begin{aligned}
-R^{\tau 1}-W^{\tau 1} & =P_{e}^{t^{\star}}-\left[U_{n d}^{\star}\right]^{\top} \int_{-1}^{1} \int_{-1}^{1}\left([B]\left[T^{\tau 1}\right]\left[\sigma^{\tau 1}\right]\right) \operatorname{det}(\mathrm{J})^{\tau 1} \mathrm{~d} \xi \mathrm{d} \eta \\
& \equiv\left[\mathrm{U}_{n d}^{\star}\right]\left[\mathrm{f}_{e}^{\tau 1}\right],
\end{aligned}
$$

where $f_{e}^{\tau 1}$ are the elementary out-of-balance forces and the vector $\sigma^{\tau 1}$ is defined:

$\left[\sigma^{\tau 1}\right]=\left[\begin{array}{lllllllll}\sigma_{11}^{\tau 1} & \sigma_{12}^{\tau 1} & \sigma_{21}^{\tau 1} & \sigma_{22}^{\tau 1} & g_{1} \varrho^{m i x, \tau 1} & g_{2} e^{m i x, \tau 1} & -m_{1}^{\tau 1} & -m_{2}^{\tau 1} & \dot{M}^{\tau 1}\end{array}\right]$,

the external virtual power $P_{e}^{t^{\star}}$ corresponds to the boundary values (for the mechanical and flow problem), except for the term related to gravity volume force, which is introduced in the $\left[\sigma^{\tau 1}\right]$ vector. By assembling all the matrices $k_{e}^{\tau 1}$ and the vectors $f_{e}^{\tau 1}$, an algebraic system is solved at each iteration to compute the correction for all the degree of freedom and to find a new test configuration of the problem.

\section{Problem at the microscopic scale}

In the framework of computational homogenization, the constitutive relations of the porous medium are derived at the Gauss

PERIODIC BOUNDARY CONDITIONS on the REV

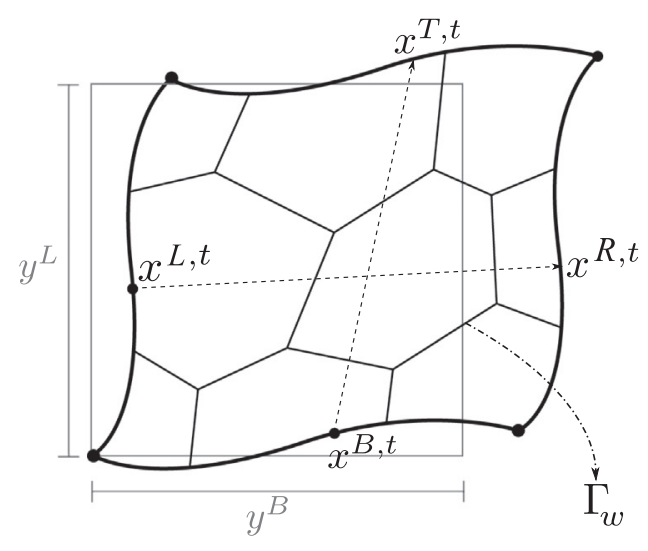

point level by solving a further finite element problem on the microstructure of the material, which characterizes a finely periodic continuum (see Fig. 1). As emphasized by Terada et al. [46] and van der Sluis et al. [47], periodic boundary conditions represent the most efficient procedure to transmit macroscopic loading on the microstructure and to compute the macroscopic constitutive relation from the solution of the microscopic problem. For this purpose, the difference of the position of two homologous points at the boundary has to meet the linear kinematical condition depicted in Fig. 2, given in terms of the macroscopic strains and the macroscopic pore pressure gradient, respectively.

Hereafter, the microscopic model formulated in Frey et al. [31] is taken into account to define the homogenized quantities listed in Eq. (10). In this model, the microstructure is characterized by hyperelastic deformable grains connected by cohesive interfaces that identify a one-dimensional network of channels $\Gamma_{w}$ in which the fluid can percolate (Fig. 2).

In the following sections, the main features of the microscopic finite element modeling are described to highlight some constitutive differences with the previous work of Frey et al. [31]. As a matter of fact, in this work the fluid is considered incompressible and the algorithm proposed for the hydraulic problem is here solved including the behavior of closed interfaces. Similarly to the macroscopic modeling, the equilibrium and the mass conservation equations have to be enforced to solve the microscopic finite element problem and to compute the quantities listed in Eq. (10). These equations are recalled in this section including an extensive description of the numerical algorithm implemented to integrate the small-scale problem. The constitutive equations of each microscopic component are also presented.

\subsection{Momentum balance equations}

The weak form of the balance of momentum for the solid phase is written in the same manner as presented in Bilbie et al. [48] and is reported as follows:

$$
\int_{\Omega^{g}} \sigma_{i j}^{g} \frac{\partial u_{i}^{g, \star}}{\partial x_{j}} d \Omega=\int_{\Lambda^{+}} t_{i}^{+} u_{i}^{g, \star+} d \Lambda^{+}+\int_{\Lambda^{-}} t_{i}^{-} u_{i}^{g, \star-} d \Lambda^{-},
$$

where $\Omega^{g}$ means the domain of the grains and $\Lambda$ the boundary of the interfaces. $\sigma_{i j}^{g}$ and $u_{i}^{g, \star}$ are respectively the Cauchy stress and the virtual displacement field of the grains, $t_{i}$ represent the forces acting on the grains due to the cohesive forces $\left(T_{t}\right.$ and $\left.T_{n}\right)$ and the fluid percolation forces $\left(p_{w}\right.$ and $\left.t_{w}\right)$ as depicted in Fig. 3(a) and (b). The effect of gravity is neglected at the microscopic scale. Both

Fig. 2. Periodic boundary conditions on the REV for the mechanical and the hydraulic problem. 
tangential $\left(t_{t}=T_{t}+t_{w}\right)$ and normal forces $\left(t_{n}=T_{n}+p_{w}\right)$ are taken into account along a given channel. The superscript notation + and - stands for the upper and lower part of the interface respectively.

Balance of momentum for the fluid phase is defined on the one-dimensional hydraulic domain $\Gamma_{w}$ and is written as follows:

$\frac{\mathrm{d} p_{w}}{\mathrm{~d} s}+t_{w}=0, \quad \forall s \in \Gamma_{w}$,

where $s$ identifies the spatial coordinate for a given channel.

To compute the pore pressure field $p_{w}$, the mass balance equations for the fluid phase have to be solved, as will be presented in the next sections. Once the field $p_{w}$ is known, the drag forces $t_{w}$ can be also computed to define the resulting forces $t_{i}:=\left(T_{t}+t_{w}, T_{n}+p_{w}\right)$.

\subsection{Mass balance equations}

The mass balance equations are prescribed for each phase of the microstructure. For the solid phase, they are automatically satisfied since an updated Lagrangian configuration is considered. For the fluid phase, the mass balance equation is enforced on the hydraulic domain $\Gamma_{w}$, which represents a one-dimensional schematization of the fluid channels defined between the solid deformable grains, Fig. 4(a). This equation means the mass flux $\bar{\omega}$ is constant along a given channel $h$ :

$\frac{\mathrm{d} \bar{\omega}^{h}}{\mathrm{~d} s}=0, \quad \forall s \in \Gamma_{w}$.

To solve the field Eq. (13) numerically, the fluid network $\Gamma_{w}$ is firstly discretized in finite elements (Fig. 4) and then, the mass balance of each node $j$ of the hydraulic domain is imposed as follows:

$\sum_{h=1}^{C} \bar{\omega}_{j}^{h}=0, \quad \forall$ node $j \in \Gamma_{w}$,

where $C$ is the total number of the channels converging in node $j$ of the fluid network. At the microscopic scale, the fluid flow is modeled by assuming a quasi static steady state in accordance with the hypothesis of quasi-static evolution of the fluid percolation process, which is consistent with the small size of the REV.

\subsection{Constitutive equations}

The behavior of each microstructural component is described with the constitutive equations presented in the following sections.

\subsubsection{Solid phase and cohesive interfaces}

The solid phase is composed of deformable solid grains which are here considered impervious. Following Frey et al. [31], the grains are regarded as isotropic, hyperelastic and undergoing finite strains (i.e. Neo-Hookean materials; see for more details [49]). The hyperelastic constitutive relation is characterized by two material properties $\lambda$ and $\mu$ (Lamé coefficients).

The cohesive forces $T_{i}$ are computed as a function of the jump in displacement $\Delta_{i}=u_{i}^{A}-u_{i}^{B}$ (Fig. 3(a)) and are assumed to be linear elastic with the following expression:

$\left[\begin{array}{c}T_{t} \\ T_{n}\end{array}\right]=\left[\begin{array}{cc}k_{t} & 0 \\ 0 & k_{n}\end{array}\right]\left[\begin{array}{c}\Delta_{t} \\ \Delta_{n}\end{array}\right]$,

$k_{t}$ and $k_{n}$ represent, respectively, the tangential and the normal stiffness of the interfaces.

\subsubsection{Fluid phase}

The assumption of incompressible fluid ( $\varrho_{w}$ is a constant) is here adopted and, the fluid flux $\bar{\omega}$ through a given channel is proportional to the gradient of the pore pressure by the conductivity $\kappa(s)$ :

$\bar{\omega}=\varrho_{w} \kappa(s)\left[\frac{\mathrm{d} p_{w}}{\mathrm{~d} s}\right]$.

The function $\kappa(s)$ depends on the viscosity $\eta$, on the shape of the channel and also on the opening of the cohesive interfaces $\Delta_{n}(s)(\kappa$ is a function of the spatial coordinate $s$ because $\left.\kappa=\kappa\left(\Delta_{n}(s)\right)=\kappa(s)\right)$. By integrating Eq. (16) with respect to the spatial coordinate $s$ of the hydraulic channel, the general expression of the flux mass $\bar{\omega}$ is obtained:

$\bar{\omega}=\varrho_{w} \frac{\left(p_{w_{j}}-p_{w_{j-1}}\right)}{\int_{j-1}^{j} \frac{1}{\kappa(s)} \mathrm{d} s}$,

$j$ and $\mathrm{j}-1$ denote the value of $s$ at the beginning and at the end of a selected channel, respectively. As a consequence of the spatial discretization of the hydraulic domain, Eq. (17) can be rewritten for each node $j$ of the fluid network using the same notation adopted by Frey et al. [31]:

$\bar{\omega}_{j}=\phi^{i}\left(\lambda_{j}-\lambda_{j-1}\right)$,

The index $i$ is referred to the $i$-th element between the nodes $j$ and $j-1$. The variables $\lambda_{j}$ and $\phi^{i}$ are defined in Table 1 , where the same quantities for a compressible fluid model [31] are also reported for comparison purposes.

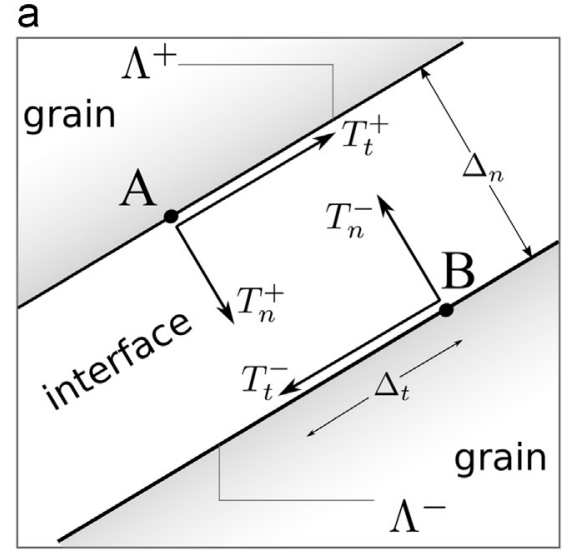

b

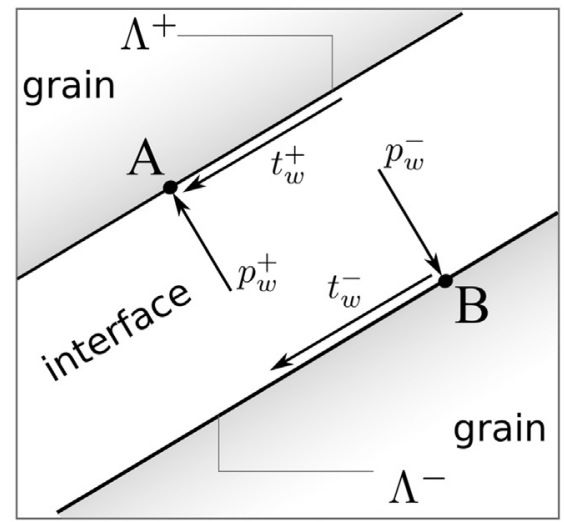

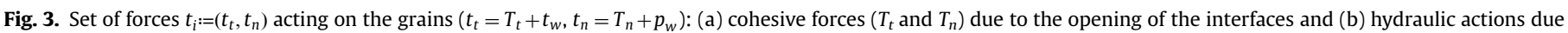
to the fluid flow $\left(t_{w}\right.$ and $\left.p_{w}\right)$. 
a

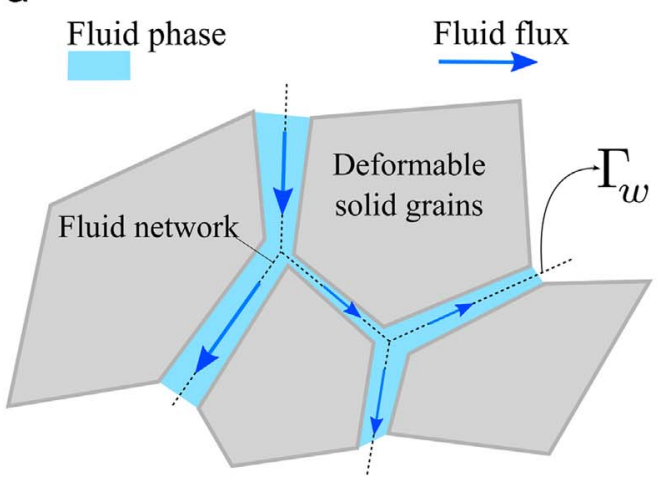

b

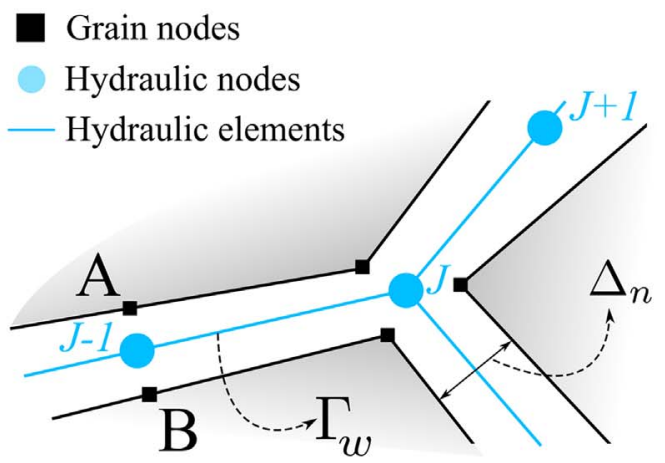

Fig. 4. Schematization of the hydraulic problem: (a) fluid flux between the interfaces and (b) discretization of the hydraulic domain $\Gamma_{w}$.

In order to give an analytical expression of the conductivity function $\kappa(s)$, an assumption to characterize the fluid flow through the intergranular space has to be introduced. Herein, the fluid percolation is modeled as a flow of a viscous fluid between two parallel plates for which an analytical solution is available, as reported in Fig. 5. Consistently with this hypothesis, the hydraulic conductivity $\kappa(S)$ is computed as follows:

$Q_{1}=\frac{\bar{\omega}}{\varrho_{w}}=\kappa(s) \frac{\mathrm{d} p_{w}}{\mathrm{~d} s}=\left[-\frac{h^{3}}{12 \eta}\right] \frac{\mathrm{d} p_{w}}{\mathrm{~d} s} \longrightarrow \kappa(s)=-\frac{h^{3}}{12 \eta}$.

By assuming the distance $h$ between the parallel plates equals to the opening of a given interface $\Delta_{n}^{i}(s)$, the function $\phi^{i}$ (Table 1 ) can be computed with numerical integration using the following expression of the hydraulic conductivity $k^{i}(s)=-\left[\Delta_{n}^{i}(s)\right]^{3} / 12 \eta$. The aforementioned hypothesis is also considered to characterize the tangential viscous forces $t_{w}$ as follows:

$t_{w}=\eta \frac{\mathrm{d} v_{1}\left(x_{2}\right)}{\mathrm{d} x_{2}}=\frac{1}{2} \frac{\mathrm{d} p_{w}}{\mathrm{~d} s} \Delta_{n}(s)$

It is worth remarking that similar assumptions on the hydraulic conductivity were also employed in other numerical methods (i.e., finite volume method [50], discrete element method [34] and lattice model [51,52]) to describe the hydromechanical behavior of soils and rocks mass. In some of these works, the hydraulic conductivity was expressed through the scheme of flow in circular tubes (i.e. Poiseuille's equation).

Table 1

Comparison of the flux mass $\bar{\omega}_{j}$ (Eq. (18)) for a rigid and a compressible fluid phase. In the latter case, $\varrho_{w}^{o}$ and $p_{w}^{o}$ represent the initial values of the fluid density and the pore pressure, respectively. $k_{w}$ is the fluid compressibility.

\begin{tabular}{ll}
\hline Rigid fluid phase & Compressible fluid phase \\
\hline$\lambda_{j}=p_{w_{j}}$ & $\lambda_{j}=\exp \left(p_{w_{j}} / k_{w}\right)$ \\
$\phi^{i}=\varrho_{w} /\left[\int_{j-1}^{j}\left(1 / \kappa^{i}(s)\right) \mathrm{d} s\right]$ & $\phi^{i}=\left(\varrho_{w}^{o} k_{w}\right) /\left[\exp \left(p_{w}^{o} / k_{w}\right) \int_{j-1}^{j}\left(1 / \kappa^{i}(s)\right) \mathrm{d} s\right]$ \\
\hline
\end{tabular}

\subsection{Numerical algorithm at the microscopic scale}

Unlike the macroscopic problem which is solved with a monolithic integration scheme, the coupled problem at the microscopic scale is dealt with using an iterative staggered algorithm as proposed by several authors in the past to simulate transient coupled problem [53,54,37].

To implement the staggered algorithm at the beginning of a given iteration $i$, the initial configuration is assumed to be known (the nodal coordinates, the corresponding opening of the interfaces and fluid pore pressure field $p_{w}$ ) and the non-linear mechanical problem is solved by considering the fluid pressure field $p_{w}$ as frozen. Once a new configuration is computed, a new opening of the interfaces $\Delta_{i}$ is obtained. Now the mechanical problem can be considered as frozen and, solving Eq. (14), a new fluid pressure field $p_{w}$ is computed. At the end of the iteration $i$, the grains composing the microstructure are submitted to a new system of forces transmitted at the interfaces: the forces $T_{t}$ and $T_{n}$ due to a new opening of the interfaces and, a new set of hydraulic forces computed in the hydraulic problem $\left(p_{w}\right.$ and $\left.t_{w}\right)$. For this reason, the mechanical problem is now unbalanced and a new iteration $i=i+1$ must be launched. This procedure is reiterated up to convergence.

It is worth noting that, in this manner, the microscopic model presents intrinsic features of coupling between the behavior of the solid and the fluid phase due to the following reasons:

(i) The variation of the pore pressure field $p_{w}$ tends to increase/ decrease the normal opening of the interfaces $\Delta_{n}$, which has an effect on the cohesive force $T_{n}$.

(ii) The conductivity function $\kappa^{i}(s)$, which determines the coefficient $\phi^{i}$ in the mass balance equations, depends on the normal opening of the interface $\Delta_{n}^{i}(s)$.

\subsubsection{Solution of the hydraulic problem}

In the staggered algorithm, the solution of the mass balance equation provides the fluid pressure field $p_{w}$ defined at each node of the hydraulic domain. For this purpose, these equations are

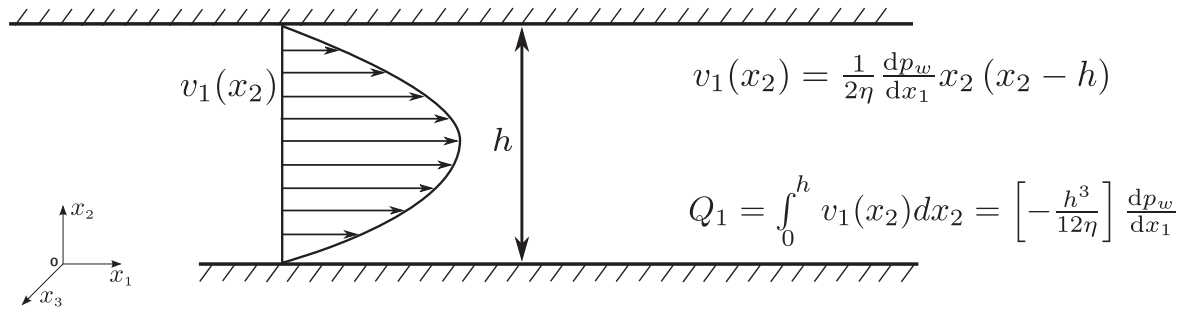

Fig. 5. Flow between two parallel plates. 
solved by computing the linear system reported in Eq. (21), which is obtained by substituting Eq. (18) into Eq. (14):

$P_{i j} \lambda_{j}=0$.

All the equations composing the matrix $P_{i j}$ are detailed in Appendix A for one of the microstructures used in Section 4 to compute a double-scale problem. To solve Eq. (21), the nonsingularity of the matrix $P_{i j}$ has to be guaranteed during the resolution process, otherwise the linear system cannot be solved with numerical tools. Such a scenario may appear in the case of closed interfaces $\left(\Delta_{n}=0\right)$ since the corresponding value of $\kappa(s)$ is equal to zero. To overcome this problem, the conductivity function $\kappa(s)$ (Eq. (19)) and the resulting viscous forces $t_{w}$ are computed with a new value of the interfaces opening $\Delta_{n}^{\text {fluid }}$ which is strictly related to the hydraulic problem:

$\Delta_{n}^{\text {fluid }}=\Delta_{n}^{i}+\Delta_{\text {min }}+\Delta_{\text {trans }} \longrightarrow k(s)=-\frac{\left(\Delta_{n}^{\text {fluid }}\right)^{3}}{12 \eta}$,

where $\Delta_{\min }$ represents a minimum value of $\Delta_{n}^{\text {fluid }}$ which enables the fluid flow to percolate even in presence of closed interfaces $\left(\Delta_{n}=0\right) . \Delta_{\text {trans }}$ corresponds to a transition value where it is still possible to consider a linear relation in the compressed regime, $\Delta_{n}^{i}<0$, between the mechanical and the hydraulic opening of the interfaces, Fig. 6(a). These two parameters $\left(\Delta_{\min }\right.$ and $\left.\Delta_{\text {trans }}\right)$ can be assumed as two new constitutive properties characterizing the flow in the network channels of the microstructure. Eq. (22) can be interpreted by virtue of the roughness characterizing the mechanical interfaces which, in the reality, are not perfectly smooth due to the asperities of the grain surface (see Fig. 6(b)). In other words, it is reasonable to assume that the fluid flow can cover alternative paths to percolate around a closed interface (i.e. $\Delta_{n}=0$ ) which is translated in Eq. (22) by introducing the parameters $\Delta_{\min }$ and $\Delta_{\text {trans }}$, Fig. 6 .

This strategy simplifies the algorithm implemented in Frey et al. [31] where, in accordance with the hypothesis of smooth interfaces, the hydraulic problem is solved excluding from Eq. (21) the compressed interfaces $\left(\Delta_{n} \leq 0\right)$ by means of a penalization method.

Even though Eq. (22) can solve the aforementioned problem, the matrix $P_{i j}$ remains singular because infinite solutions can be computed with the hydraulic boundary conditions shown in Fig. 2. Indeed, one additional constraint on the average pressure has to be included to restore the well-posedness of the matrix $P_{i j}$. This constraint is enforced by means of penalizing one nodal pressure, which is then updated. In the computations presented in Section 4, the pore pressure of node $2\left(j=2\right.$ in the hydraulic domain $\Gamma_{w}$, see for instance Fig. A1(b)) has been fixed and initially enforced to be equal to the macroscopic pressure $p^{\text {macro }}$ computed on the integration point level of the large-scale problem. By using this strategy, the non-singularity and consequently the uniqueness of the solution of the matrix $P_{i j}$ is restored. It is worth highlighting that the solution of the algorithm does not depend on the particular node selected to enforce $p^{\text {macro }}$ (i.e., the node 2 ) since the convergence criterium is prescribed in terms of average of the pore pressure.

The mass balance equation (Eq. (14)) is strongly non-linear if the fluid is modeled as compressible and, for this reason, an iterative algorithm is implemented in Frey et al. [31]. This algorithm, here recalled in Table 2, is adopted to compute the pressure field for a rigid fluid. In such a case, the problem is linear in the unknown $p_{w}$ and the microscopic fluid pressure field is computed with a single iteration.

\section{Table 2}

Algorithm solving the hydraulic problem.

- The normal opening of the interfaces $\Delta_{n}{ }^{i}$ are given at the iteration $i$ of the mechanical problem

1. Initialization of the fluid system:

(a) Computation of $\Delta^{\text {fluid }}{ }_{n}$ : $\Delta^{\text {fluid }}{ }_{n}=\Delta_{n}^{i}+\Delta_{\text {min }}+\Delta_{\text {trans }}$

(b) Computation of $k(s)^{h}$ and $\int \frac{1}{k(s)^{h}} d s$ for each fluid channel h by using the opening of the interface $\Delta_{n}$ of each interface (Eq. (19)). (c) Initialization of $P_{i j}$ with the macro pore pressure at the node 2 : $p_{w_{2}}=p^{\text {macro }}$

(d) Computation of the $\phi_{i}$ for each fluid channel

(e) Assembling of matrix $P_{i j}$ with all terms $\phi^{h}$ and with the periodic boundary conditions

2. Iterative procedure: iteration $k=1$

(a) Iteration $k=k+1$

- If $k=1 \Longrightarrow$ go to (2b)

- Else if $k>1 \Longrightarrow$ updating the matrix $P_{i j}$ with $p_{w_{2}}$

(b) Computation of $p_{w_{i}}$ solving the linear system $P_{i j}$

(c) Computation of tangential viscous forces $t_{w}$

(d) Computation of average value of the field pressure in the channel:

$p_{w}^{a v}=\sum p_{w_{i}} / \Omega_{w}$

(e) Computation of the difference: $\Delta p_{w}=p^{\text {macro }}-p_{w}^{a v}$.

(f) Updating of the average pressure: $p_{w_{2}}=p_{w_{2}}+\Delta p_{w}$

(g) Convergence test:

- If $\Delta p_{w} \leq$ tol $l^{\text {fluid }} \Longrightarrow$ go to 3

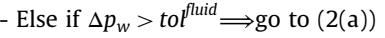

3. End of the algorithm solving the fluid system

- Updating the interfaces forces of the mechanical system with the

fluid forces $\left(t_{w}\right.$ and $\left.p_{w}\right)$. A new iteration $i=i+1$ can be computed. a

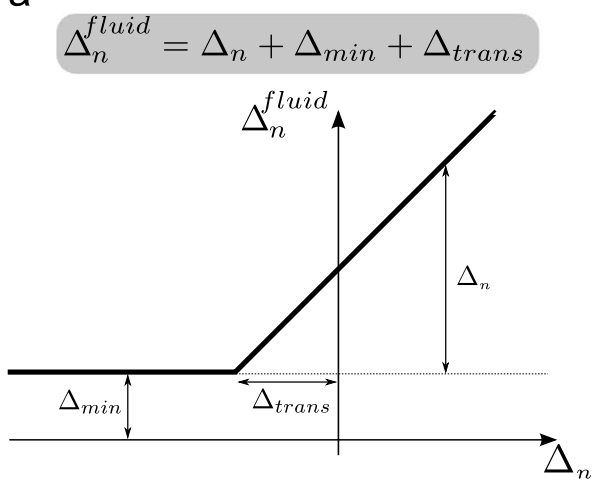

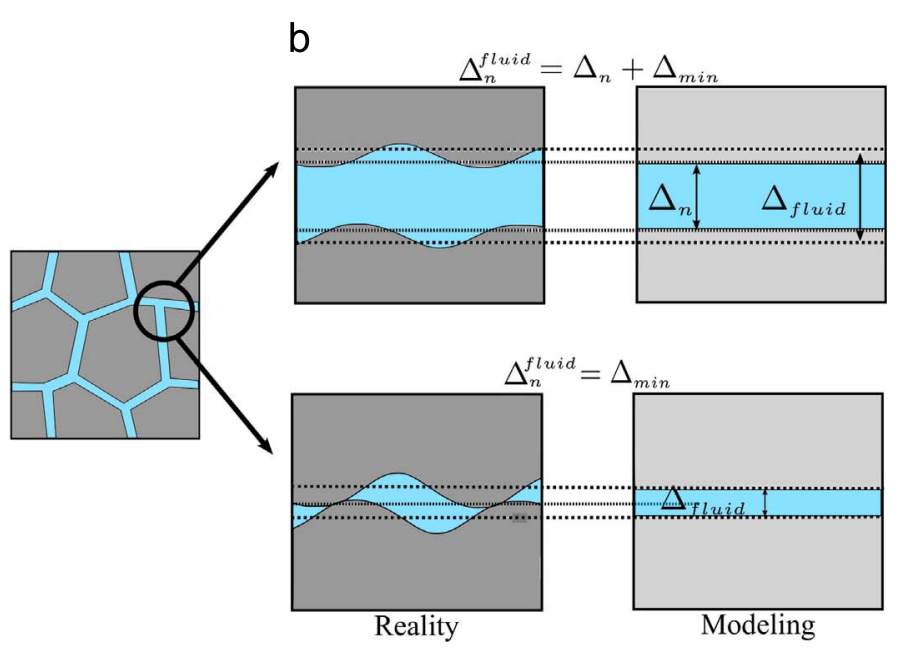

b

Modeling

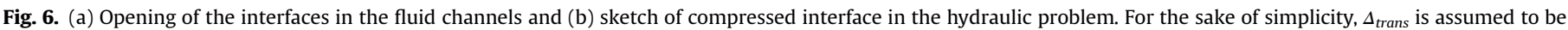
equal to zero. 


\subsection{Macroscopic behavior of the mixture}

In the framework of $\mathrm{FE}^{2}$ method, the quantities listed in Eq. (10) are obtained with a numerical operation of averaging on the microscopic fields as follows:

- The total stress of the mixture $\sigma_{i j}{ }^{m i x}$ :

$\sigma_{i j}^{m i x}=\frac{1}{\Omega}\left(\int_{\Omega_{s}} \sigma_{i j}^{s} d A+\int_{\Omega_{w}} p_{w} \Delta_{i j} d A\right)$,

$\Omega_{s}$ and $\Omega_{w}$ are the total surfaces of the grains and of the hydraulic interfaces, respectively. $\Omega$ is the total surface of the microstructure, (i.e. $\Omega=\Omega_{s}+\Omega_{w}$ ). Eq. (23) shows an important outcome of the computational homogenization approach since the Cauchy stress of the mixture is a result of an upscaling operation on the coupled behavior of the microstructure. Consequently, the classical concept of effective stress, whose definition can be arguable, is not anymore necessary.

- The homogenized value of the density:

$$
\varrho^{m i x}=\frac{M_{s}+M_{w}}{\Omega}=\frac{1}{\Omega}\left(\varrho_{s} \Omega_{s}+\int_{\Omega_{w}} \varrho_{w} d A\right),
$$

$\varrho_{s}$ and $\varrho_{w}$ represent, respectively, the density of the solid and the fluid phases. $\Omega$ is the REV domain.

- The variation of the fluid mass content:

$$
M_{w}=\frac{1}{\Omega} \int_{\Omega_{w}} \varrho_{w} d A \quad \dot{M}_{w}=\frac{M_{w}^{t}-M_{w}^{t+\Delta t}}{\Delta t} .
$$

It is important to remark that the fluid mass content is here influenced by two aspects: the opening of the interfaces and the compressibility of the fluid. Since in this work the fluid is considered incompressible, only the opening of the interfaces affects this quantity.

- The fluid mass flux:

$m_{x}^{m i x}=\frac{1}{\bar{a}} \sum_{j=1}^{A} \bar{\omega}_{x_{j}} \quad m_{y}^{m i x}=\frac{1}{\bar{a}} \sum_{k=1}^{B} \bar{\omega}_{y_{k}}$,

A and $B$ represent the number of the nodes of the network channels on the boundary at the right and at the top of the microstructure, respectively. $\bar{a}$ is the size of the REV.

\section{Validation of the model: comparison with Biot's theory}

To validate the implementation of the presented model in the finite element code Lagamine (code of University of Liège, Belgium, [32,33]), the general theory of consolidation formulated by Biot [1] is considered. In this study, a consolidation test is performed and the numerical results are compared with the onedimensional analytical solution for the corresponding Biot-like a

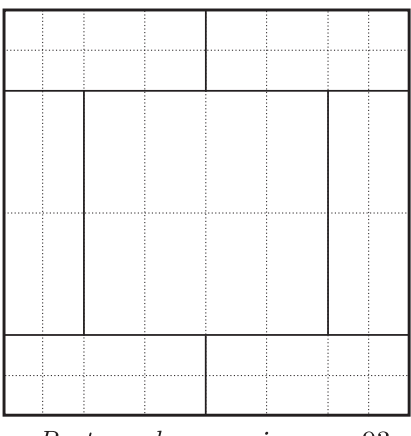

Rectangular grains:

93

nodes, 72 finite elements. b

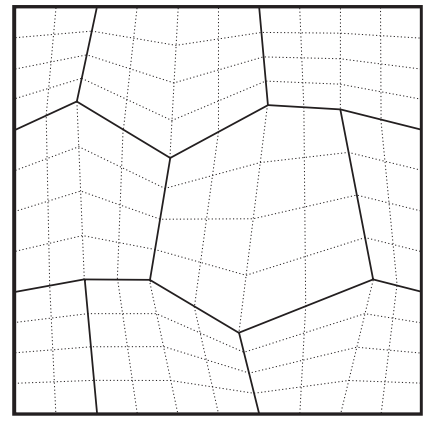

Irregular grains: 195 nodes,

164 finite elements.

Fig. 8. Microstructures used in the numerical tests. The dotted lines indicate the spatial discretization in finite elements and the thick lines identify the boundaries of the grains.

Table 3

Hydromechanical parameters for each component of the microstructure.

\begin{tabular}{lllllllll}
\hline \multicolumn{1}{l}{ Grains } & \multicolumn{4}{c}{ Cohesive interfaces } & \multicolumn{2}{l}{ Fluid phase } \\
\hline$\lambda$ & 960.0 & $\mathrm{MPa}$ & $\kappa_{t}$ & 30.0 & $\mathrm{MPa}$ & $\nu$ & $10^{-9}$ & $\mathrm{MPa} \mathrm{s}$ \\
$\mu$ & 1440.0 & $\mathrm{MPa}$ & $\kappa_{n}$ & 30.0 & $\mathrm{MPa}$ & $\varrho_{w}$ & $10^{-3}$ & $\mathrm{~g} / \mathrm{mm}^{3}$ \\
$\varrho_{s}$ & $2.7 \times 10^{-3}$ & $\mathrm{~g} / \mathrm{mm}^{3}$ & & & & $k_{w}$ & $2.2 * 10^{3}$ & $\mathrm{MPa}$ \\
& & & & & $\Delta_{\text {min }}$ & 0 & $\mathrm{~mm}$ \\
& & & & & & $\Delta_{\text {trans }}$ & 0.05 & $\mathrm{~mm}$ \\
\hline
\end{tabular}

porous medium. The boundary conditions of the computed numerical problem are schematized in Fig. 7.

The loading $q$ is kept constant during the test and is equal to 0.1 MPa. The macroscopic domain depicted in Fig. 7 is here discretized with a column of 1000 finite elements; 2D squared isoparametric elements with eight nodes for the displacement and the pore pressure fields have been employed for the presented computations. In this study, the effects due to the gravity are neglected. In the following sections, the macroscopic behavior of the material is modeled by considering two different microstructures $(\bar{a}=1 \mathrm{~mm})$ : the first one is composed by rectangular grains and the second one by irregular grains (Fig. 8). In both cases, isoparametric elements with four nodes are used to discretize the domain composed by the elastic grains. The parameters used to model each component of the microstructures plotted in Fig. 8 are reported in Table 3 and are considered the same for all the elastic grains and the cohesive interfaces.

The homogenized hydromechanical properties of the computed porous medium are extracted from the local stiffness matrix $E^{\tau 1}$ (Eq. (7)) which, for a one-dimensional problem $\left(u_{1}=0\right.$ and

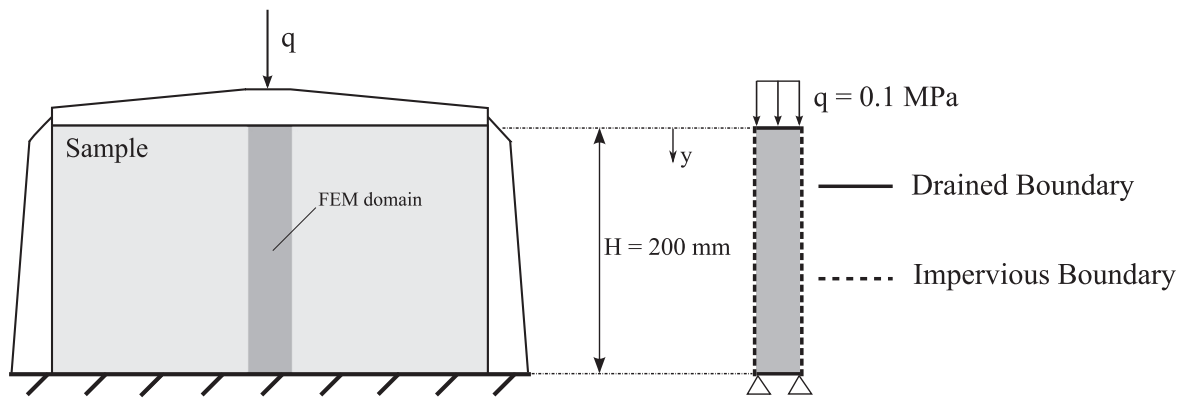

Fig. 7. Finite element domain and initial boundary conditions of the consolidation problem. 


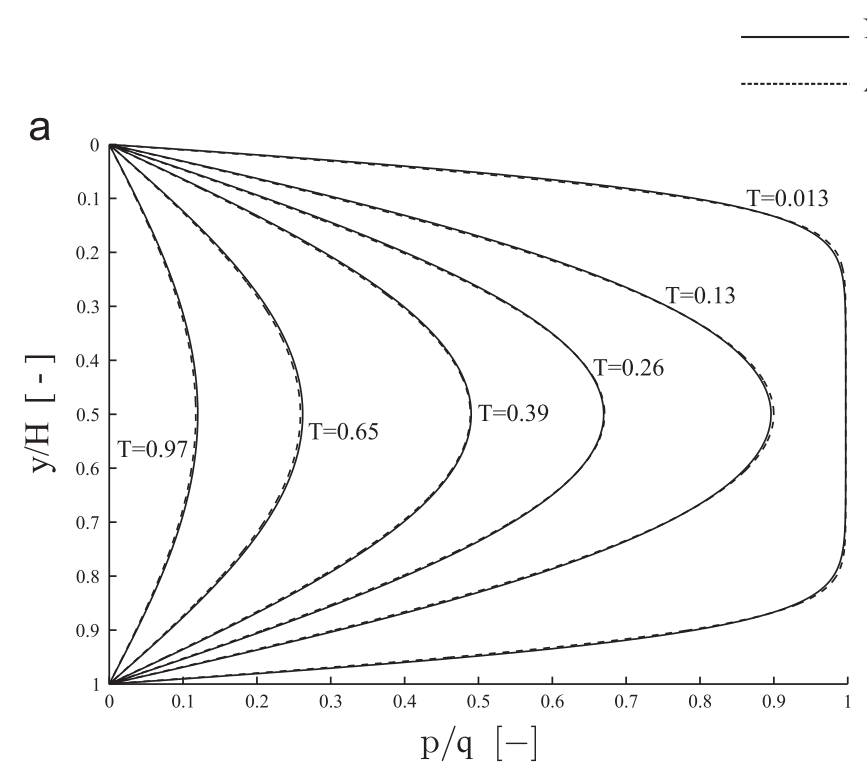

Numerical

Analytical

Fig. 9. Comparison between the numerical results and the general theory of consolidation [1]: (a) normalized pore pressure and (b) average degree of consolidation $U$.

$\left.\partial(\cdot) / \partial x_{1}=0\right)$, can be simplified as follows:

$\left[E^{\tau 1}\right]=\left[\begin{array}{ccc}C_{2222} & B_{222} & A_{22} \\ K_{222} & N_{22} & L_{2} \\ H_{22} & J_{2} & F\end{array}\right]$,

$C_{2222}$ corresponds to the oedometric Young modulus $E_{o e}$ and $A_{22}$ is the Biot coefficient $b . N_{22} / \varrho_{w}$ and $F / \varrho_{w}$ represent the hydraulic permeability $K$ and the storage mass coefficient $1 / Q$, respectively. The other terms of Eq. (27) represent the remaining coupling properties between the mechanical and the hydraulic problem and they will be discussed later.

Hereafter, the presented numerical results are performed by considering two convergence criteria based on an Euclidean norm for the nodal displacement corrections and the non-equilibrated nodal forces. The iterative procedure of the macroscopic problem stops when both convergence criteria are less than a given tolerance. For the displacement and for the force criteria, the selected tolerances tol $_{d}$ and tol $_{f}$ are equal to tol $_{d}=t_{0} l_{f}=10^{-5}$.

To solve the staggered algorithm at the microscopic observation scale, the same kind of criterion is taken into account and the tolerances are set to tol $l_{\text {micro }}=10^{-6}$ and to to fluid $=10^{-7}$ for the mechanical and the hydraulic problem, respectively (see Table 2). By using the aforementioned constitutive equations and the selected parameters shown in Table 3, the numerical behavior of the staggered algorithm is stable and the microscopic problem converges with 2-3 iterations.

\subsection{Microstructure with rectangular grains}

The microstructure presented in Fig. 8(a) is here considered to solve the initial boundary value problem schematized in Fig. 7. The numerical results are compared with the analytical solution in Fig. 9, where the spatial trend of the normalized pore pressure $p / q$ and the average degree of consolidation $U$ are plotted to describe the consolidation process. In this figure it is possible to observe that the computed solution is in good agreement with the solution referred to the classical theory of Biot [1] and, as a result, the homogenized hydromechanical behavior of this microscopic model reproduces a Biot-like porous medium at the macroscopic scale.

The analytical solution plotted in Fig. 9 is expressed as a function of the consolidation coefficient $c_{v}$ and the time factor $T=t c_{v} /(H / 2)^{2}$, where $t$ is the physical time and $H$ is the vertical

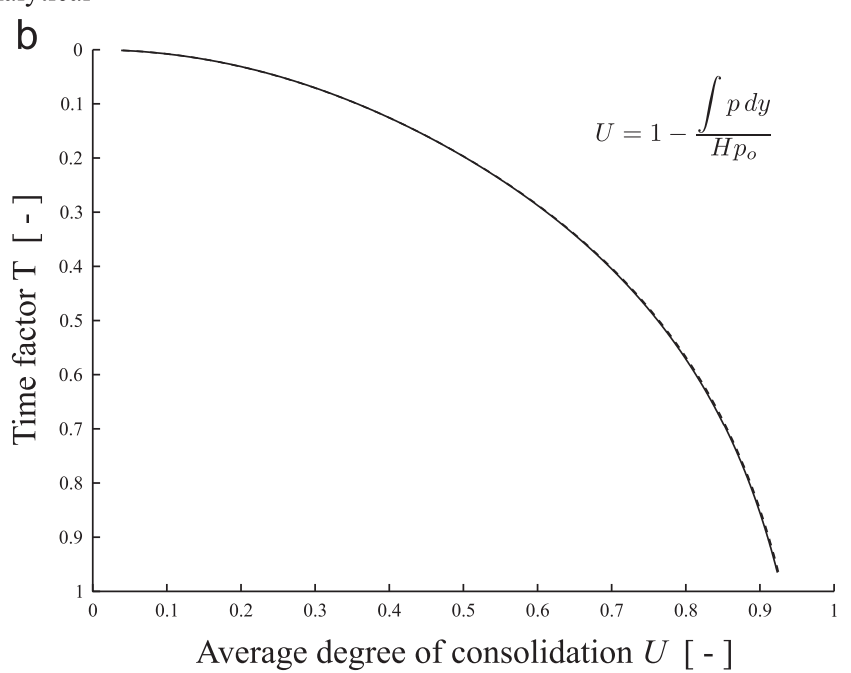

size of sample. The consolidation coefficient $c_{v}$ is defined in [1] and it is reported as follows:

$c_{v}=\left[\frac{1}{K}\left(\frac{b^{2}}{E_{o e}}+Q\right)\right]^{-1}$

In order to calculate the coefficient $c_{v}$ and to detail the behavior of the equivalent porous medium, the hydromechanical properties for different states of the consolidation process are plotted in Fig. 10. In this figure, the homogenized parameters show a nonlinear trend due to an intrinsic coupling between the skeleton and the fluid phase of the microstructure. The corresponding variability of each parameter during the test can be calculated from Fig. 10; for this case study, the Biot coefficient $b$ and the stored mass coefficient $1 / Q$ are characterized by small variations between their maximum and minimum values (less than $0.02 \%$ ) which can be assumed negligible. The same observation may be applied for the homogenized oedometric Young's modulus $E_{o e}$ even though the variability of this parameter is bigger (around $1 \%$ ) with respect to the coefficients $b$ and $1 / Q$. In this computation, the hydraulic permeability $K$ is the parameter most affected by the intrinsic microstructural coupling and its variability is around $8 \%$. Since the theory of Biot [1] is formulated by assuming constant hydromechanical properties, the consolidation coefficient $c_{v}$ (Eq. (28)), used in the analytical solution, is here calculated by performing an average between the maximum and the minimum value of each parameter during the consolidation process. It is interesting to remark that, for the given set of microstructural parameters, the spatial trend of the Biot's coefficient (Fig. 10(b)) is close to one. This result indicates that, in accordance with the Terzaghi's theory, the low deformability of the elastic grains leads to the approximation of a rigid solid phase.

As can be observed in Eq. (27), the local stiffness matrix is also composed of other terms which are representative of further coupling mechanisms between the mechanical and the hydraulic problem. The coefficient $B_{222}$ describes the coupling between the mechanical response and a given value of $\partial \mathrm{d} p / \partial x_{2}$; this quantity represents the macroscopic effect of the tangential viscous forces $t_{w}$ in the microscopic problem and, for this case study, due to small values of the flux mass $\bar{\omega}_{j}$, the homogenized parameter $B_{222}$ is close to zero. The same result is here obtained for the coefficients $J_{2}$ and $L_{2}$. The former coefficient is close to zero because the fluid mass content depends on the normal opening of the interfaces, 

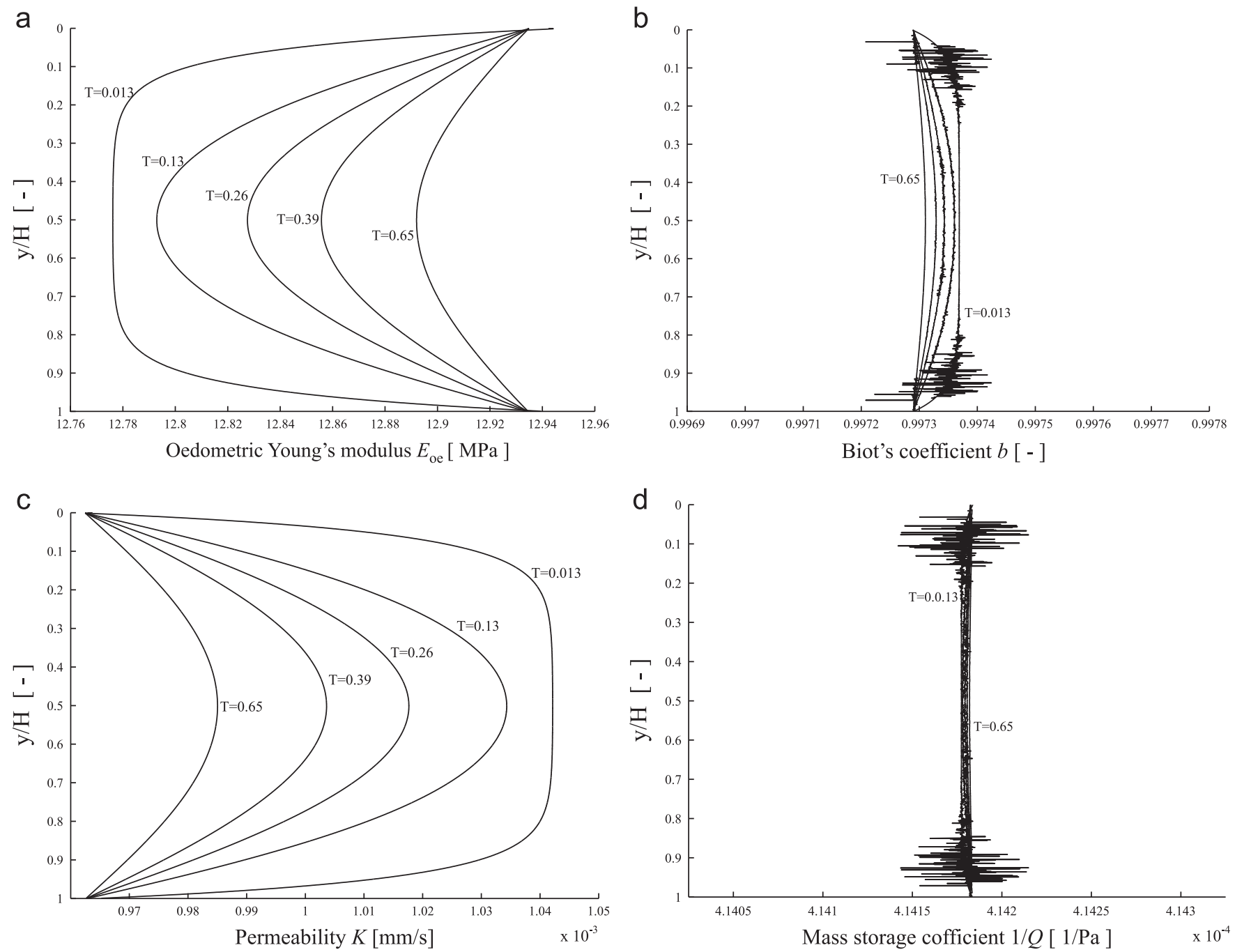

Fig. 10. Homegenized hydromechanical parameters with the microstructure composed by rectangular grains: (a) Young modulus $E_{o e}$, (b) Biot coefficient $b$, (c) permeability $K$, and (d) stored fluid coefficient $1 / Q$.
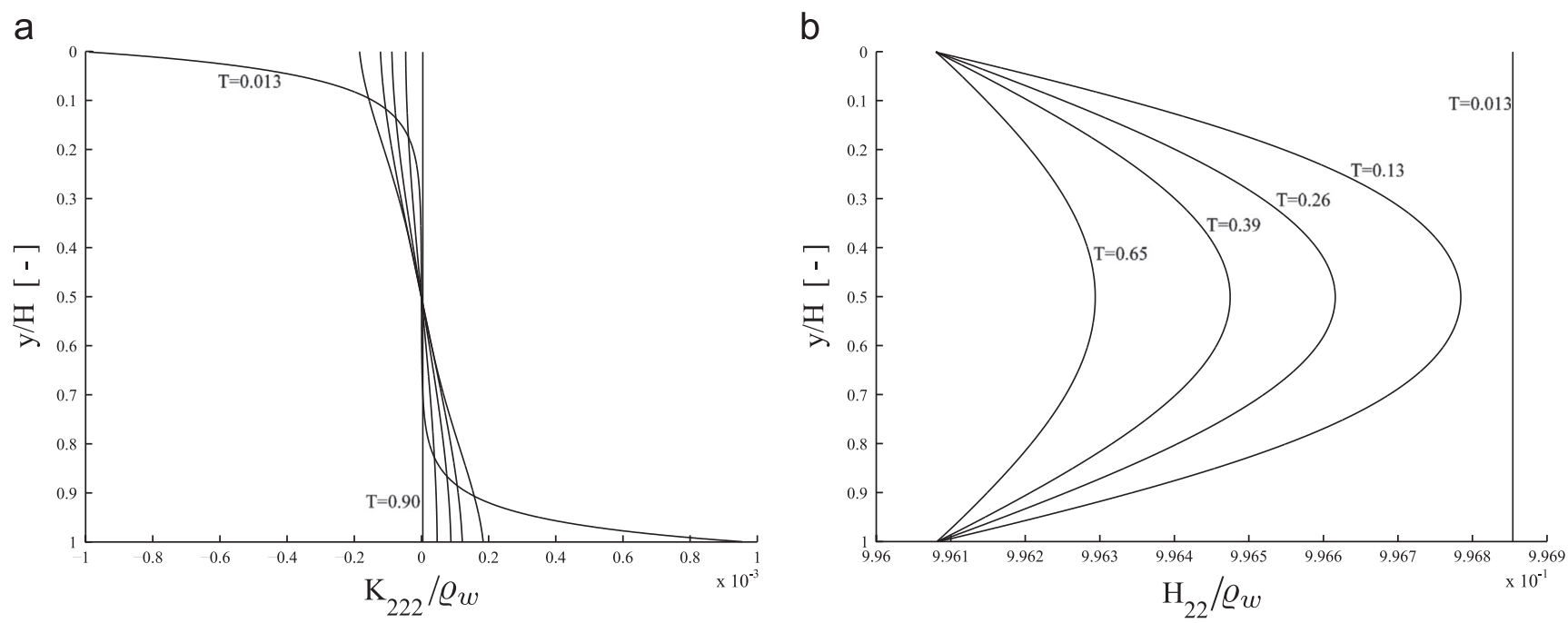

Fig. 11. Hydromechanical coefficients $K_{222} / \varrho_{w}$ and $H_{22} / \varrho_{w}$.

whose deformed configuration is mostly influenced by the macroscopic quantities $\mathrm{d} p$ and $\partial \mathrm{d} u_{2} / \partial x_{2}$ enforced on each microstructure; the latter coefficient is close to zero because an increment of the pore pressure does not affect considerably the flow process for a compressed microstructure.

The other two homogenized parameters, $H_{22} / \varrho_{w}$ and $K_{222} / \varrho_{w}$ are plotted in Fig. 11 and they couple the hydraulic response for a 

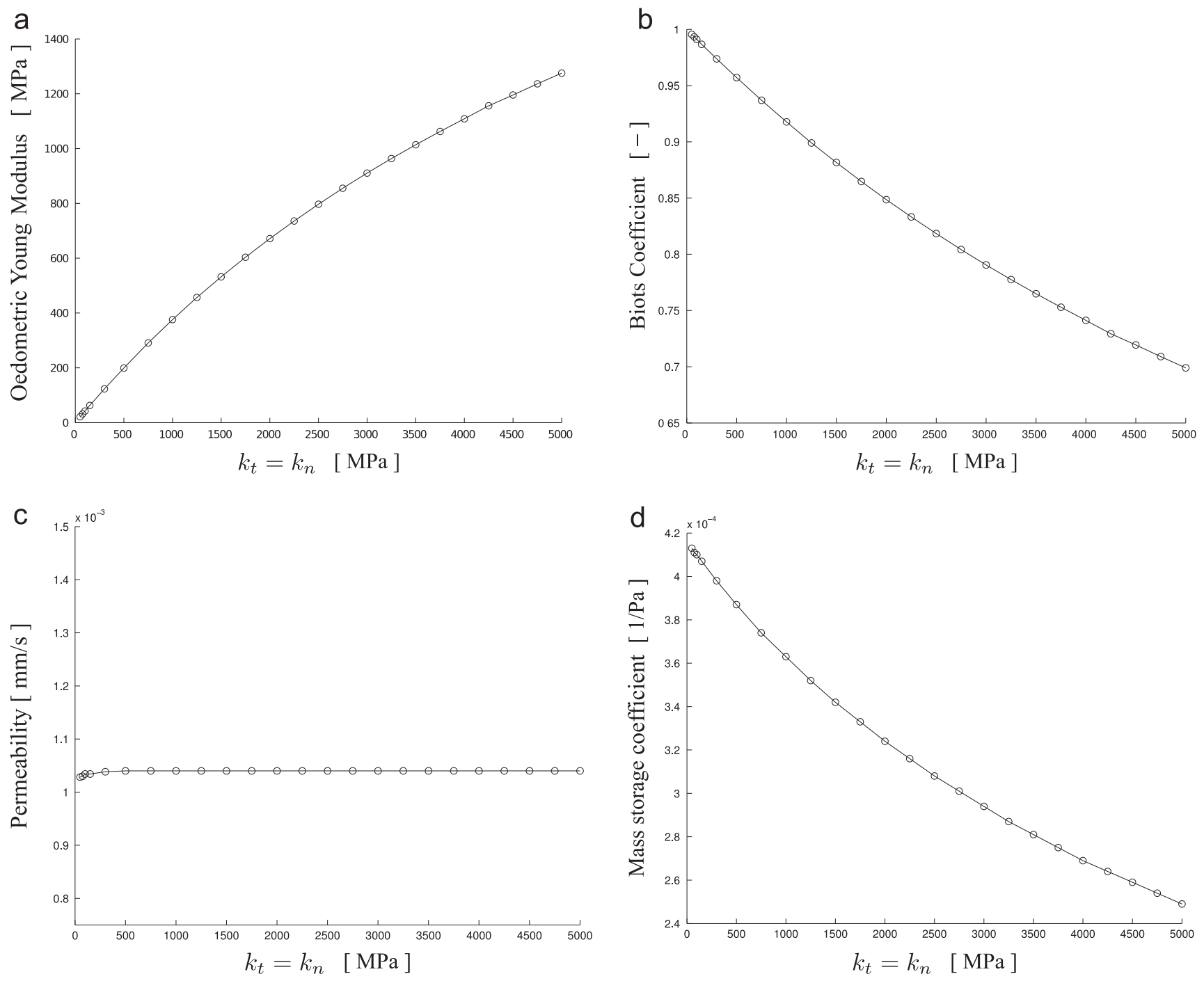

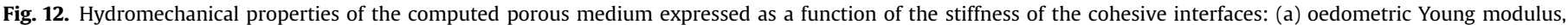
(b) Biot's coefficient, and (c) mass storage coefficient.

given increment of strain. The parameter $\mathrm{H}_{22} / \varrho_{w}$ is close to the value of the Biot coefficient $b$ shown in Fig. 10(b) consistently with the theoretical formulation of Biot [1]. For the computed problem, the component $K_{222} / \varrho_{w}$ has an effect at the beginning of the consolidation process but rapidly tends to a negligible value since the dissipation of the pore pressure involves more compressed interfaces. A microscopic interpretation of this initial boundary value problem is given in Fig. 15, where the interface forces and the pore pressure field are plotted for one microstructure of the macroscopic domain.

In order to better emphasize how the deformability of the grains can affect the mechanical properties of the equivalent porous medium, some computations have been performed keeping the parameters of Table 3 with the only exception of the stiffness $k_{t}$ and $k_{n}$ of the cohesive interfaces, which now are higher. Due to an increase of $k_{t}$ and $k_{n}$, higher contact forces are generated between the elastic grains which undergo a more significant strain field. The more deformable the elastic grains are at the microscopic scale, the lower the Biot's coefficient is at the macroscopic scale (Fig. 12(b)). The same effect is obtained by decreasing the stiffness of the elastic grains and, for this reason, it is not plotted here. Furthermore, an increase of the interface stiffness has an effect also on the homogenized oedometric Young modulus which tends to increase (Fig. 12(a)). The stored mass coefficient $1 / Q$, as a function of $k_{t}=k_{n}$, is plotted in Fig. 12(d) and it shows a similar trend to the Biot coefficient. As a matter of fact, an increase of the stiffness $k_{t}=k_{n}$ makes the opening of the interfaces smaller, reducing thereby the content of stored fluid. Accordingly, the mass storage coefficient $1 / Q$ decreases. For the same reason (i.e. a small variation of $\Delta_{n}^{i}$ produced by an increasing stiffness $k_{t}=k_{n}$ ) the macroscopic permeability $K$ is mainly governed by the parameter $\Delta_{\text {trans }}$ and is constant for the whole range of the stiffness values, as shown in Fig. 12(c). This effect is further accentuated by the cubic dependency between $\kappa(s)$ and $\Delta_{n}^{\text {fluid }}$ (Eq. (22)).

It is worth highlighting that the non-linear behavior of the grains described by finite elasticity [49] is triggered for high values of the stiffness of the cohesive interface. This behavior is reflected on the homogenized coefficients of the mixture plotted in Fig. 12 which start to show a significant non-linear trend for $k_{t}=k_{n}>750 \mathrm{MPa}$. Indeed, at the macroscopic scale, the corresponding effect on the consolidation process is shown in Fig. 13 for different values of $k_{t}$ and $k_{n}$. In these figures, it is possible to observe that, at the beginning of the consolidation process, the pore pressure profile is no longer close to the external loading $q$ because a portion of the loading is sustained by the solid skeleton. This effect, consistently with the Biot theory, is more important for smaller Biot coefficients. 


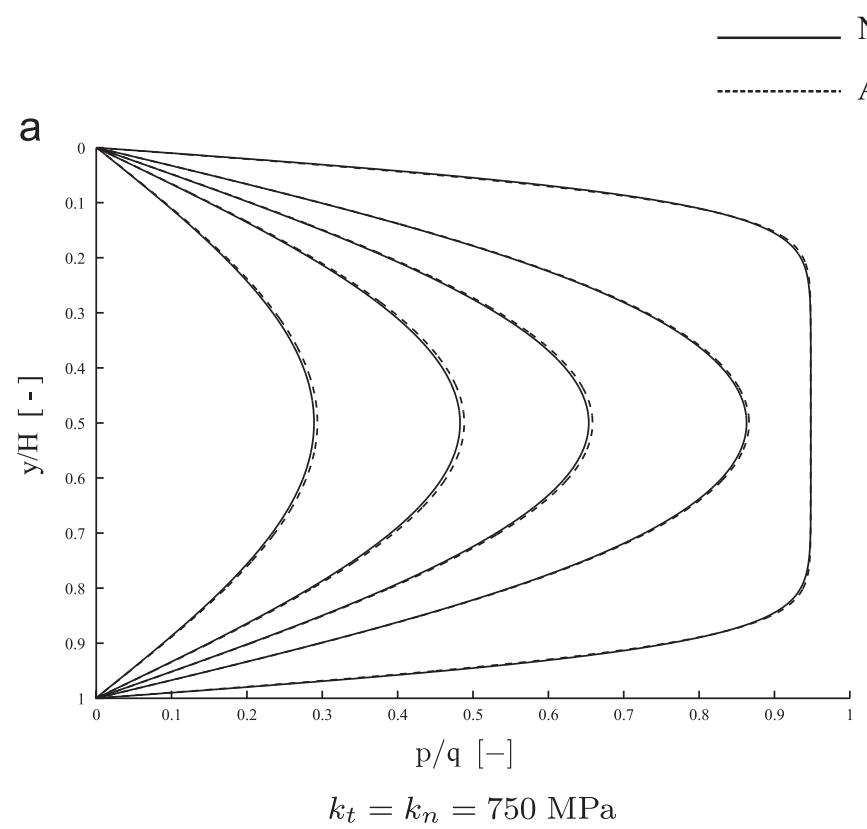

\section{Numerical}

Analytical

b
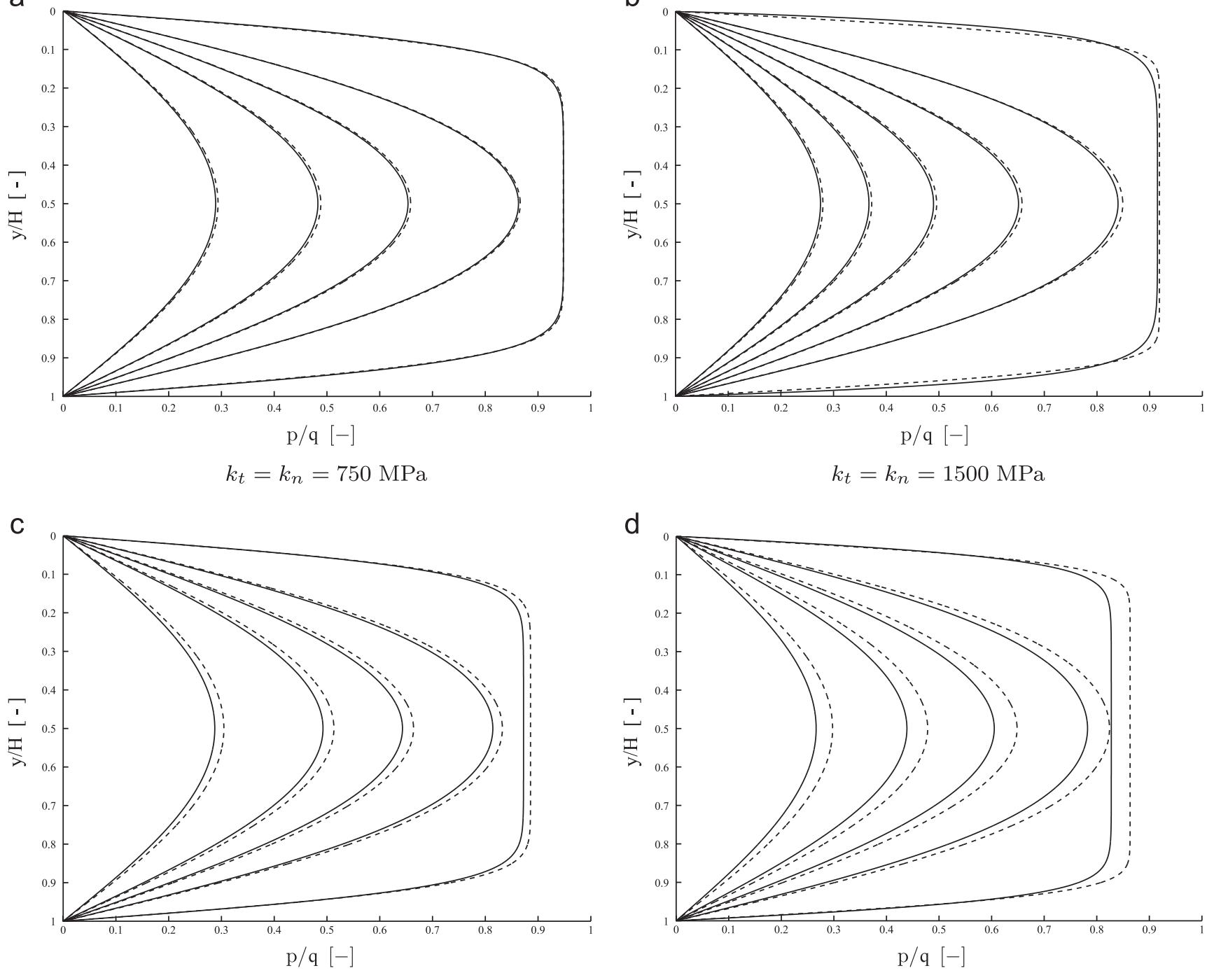

$k_{t}=k_{n}=1500 \mathrm{MPa}$

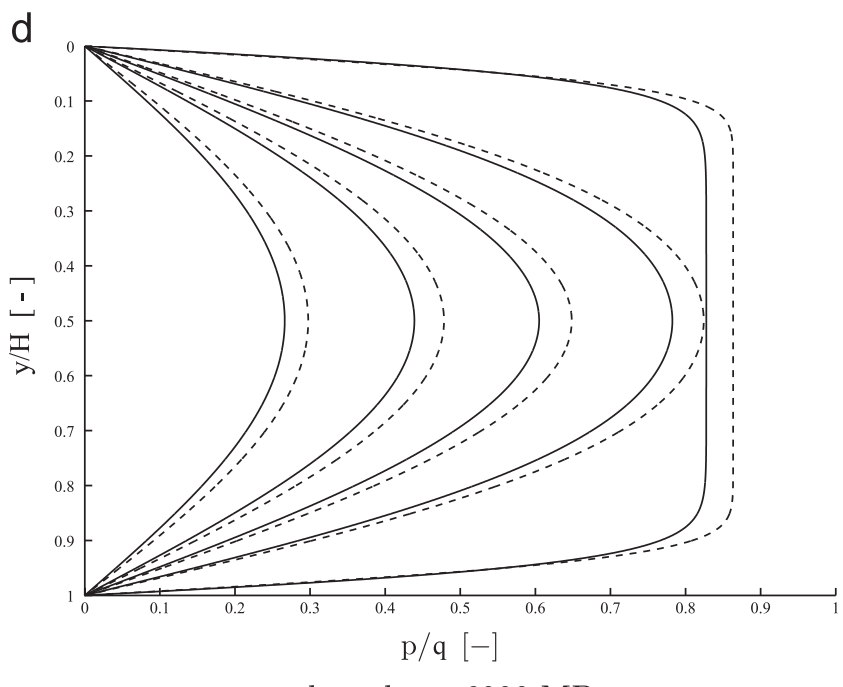

$k_{t}=k_{n}=3000 \mathrm{MPa}$

$k_{t}=k_{n}=6000 \mathrm{MPa}$

Fig. 13. Comparison between the numerical results and the analytical solution for different values of the stiffness $k_{t}=k_{n}$ of the cohesive forces.

As a result, the mismatch between the numerical and the analytical solution, observed in Figs. 13, demonstrates a further macroscopic effect related to the non-linear behavior of the mixture for high values of $k_{t}$ and $k_{n}$. Even though this mismatch is more accentuated for increasing stiffness of the interfaces, the qualitative trend of the consolidation process is conserved.

\subsubsection{Convergence analysis}

In the framework of finite element computations, the definition of the consistent stiffness matrix $E_{i j}^{\tau 1}$ (Eq. (7)) can play an important role on the iterative process to solve the time step problem. As mentioned in Section 2.2, here the consistent stiffness matrix is calculated with a classical perturbation method which represents a numerical way to compute a derivative. In this method the $j$-th column of $E_{i j}^{\tau 1}$ is computed as follows:

$E_{i j}^{\tau 1}=\frac{\sigma_{i}\left(\mathrm{~d} U_{j}^{\tau 1}\right)-\bar{\sigma}_{i}\left(\mathrm{~d} U_{j}^{\tau 1}+\xi_{k}\right)}{\xi}$.

The vector $\sigma_{i}$ represents the quantities listed in Eq. (10) and is calculated with the kinematic $\mathrm{d} U^{\tau 1}$ (Eq. (6)). $\bar{\sigma}_{i}$ has the same meaning of $\sigma_{i}$ but it is computed with a perturbated kinematic $\left(\mathrm{d} U_{j}^{\tau 1}+\xi_{k}\right) \cdot \xi_{k}$ is defined as $\xi_{k}=\xi \mathcal{T}_{k}$ where the $k$-th component of $\mathcal{T}_{k}$ is equal to one if $k=j$ and zero otherwise. $\xi$ is the perturbation of the problem. The main drawback of this method is the choice of $\xi$ which can considerably affect the convergence rate of the problem. Indeed, if the perturbation $\xi$ is too small, the computed stiffness matrix can be influenced by the numerical noise since the corresponding component of $E_{i j}^{\tau 1}$ is computed as a difference. On the other hand, if the perturbation $\xi$ is too big, the components of the matrix $E^{\tau 1}$ are far from their theoretical values.

For these reasons, the parameter $\xi$ has to be tested to select a given value which guarantees a good performance of the convergence process. In fact, a sensitivity study has been performed for the consolidation problem and a quadratic convergence has been obtained for a range of perturbations $\xi$ between $\xi=0.1$ and $\xi=10^{-7}$. In the end, the numerical solutions computed for the microstructure with rectangular grains are carried out with a perturbation $\xi$ equal to $\xi=10^{-4}$. For this case study, the iteration number related to a converged solution is constant for all the time steps and is equal to three iterations. The convergence trend is 
plotted in Fig. 14 for a particular step corresponding to the time factor $T=0.13$. In this figure, the computation has been repeated with lower tolerances $\left(\right.$ tol $_{d}=$ tol $_{f}=10^{-20}$ ), much lower than the numerical precision provided by the computer, giving also an insight into the numerical noise of the selected problem. It is interesting to note that, due to the limited variability of the parameters $b$ and $Q$ some oscillations can be observed in Fig. 10 (b) and (d) as a consequence of the small value of the perturbation

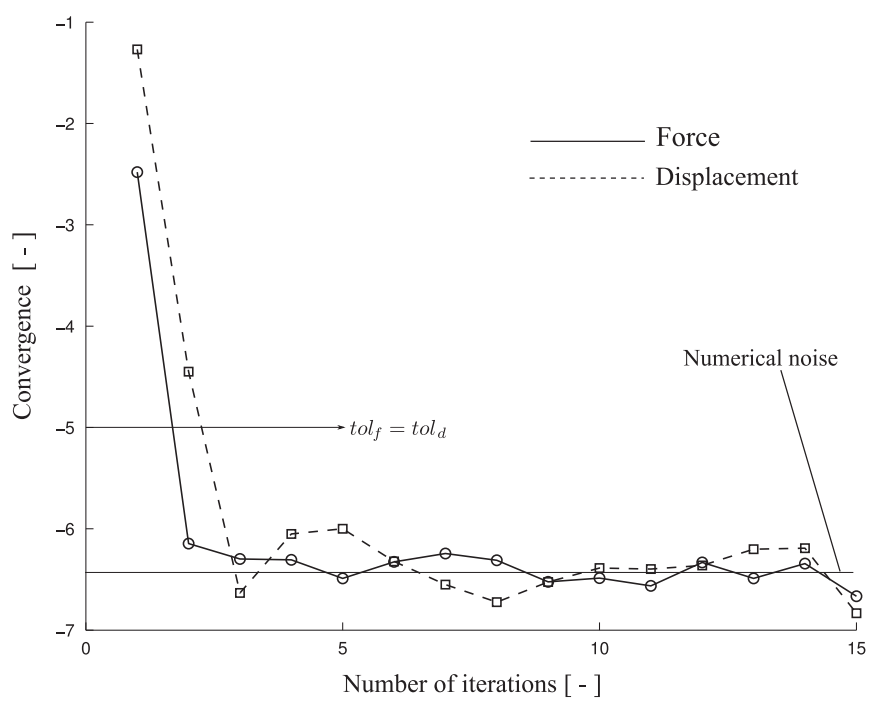

Fig. 14. Convergence of the macroscopic problem: logarithm of the convergence norms (in force and in displacement). $\xi$. As a matter of fact, the perturbation method should be optimized by computing each column of the matrix $E^{\tau 1}$ with different values of $\xi$. This optimization has not been implemented in this work since, even though small oscillations appear, a good convergence rate is reported.

\subsection{Microstructure with irregular grains}

In order to confirm the results obtained with the microstructure of Fig. 8(a), the same initial boundary value problem is now computed with the microstructure composed of irregular grains, Fig. 8(b) and the micromechanical properties presented in Table 3. The comparison between the analytical and the numerical solution is plotted in Fig. 16(a) and (b) and, also for this case, the results prove that the computed equivalent porous medium reproduces the qualitative behavior of a Biot-like porous medium.

The homogenized oedometric Young's modulus $E_{o e}$ and the permeability $K$ are shown in Fig. 17 and present a similar trend like the case computed in Section 4.1. Even though the order of magnitude is the same, the oedometric Young's modulus $E_{o e}$ and the permeability $K$ are characterized by different values with respect to the microstructure with rectangular grains, indicating that the change in geometry of the microstructure has an effect on the hydromechanical properties of the corresponding equivalent porous medium. The other homogenized parameters are not shown here since they are, with a good approximation, close to the values presented for the microstructure with rectangular grains. From a more general viewpoint, it is important to remark that the shape of the grains plays a crucial role to replicate material symmetries which can characterize different types of anisotropic behavior

\section{MICROSCOPIC SCALE}

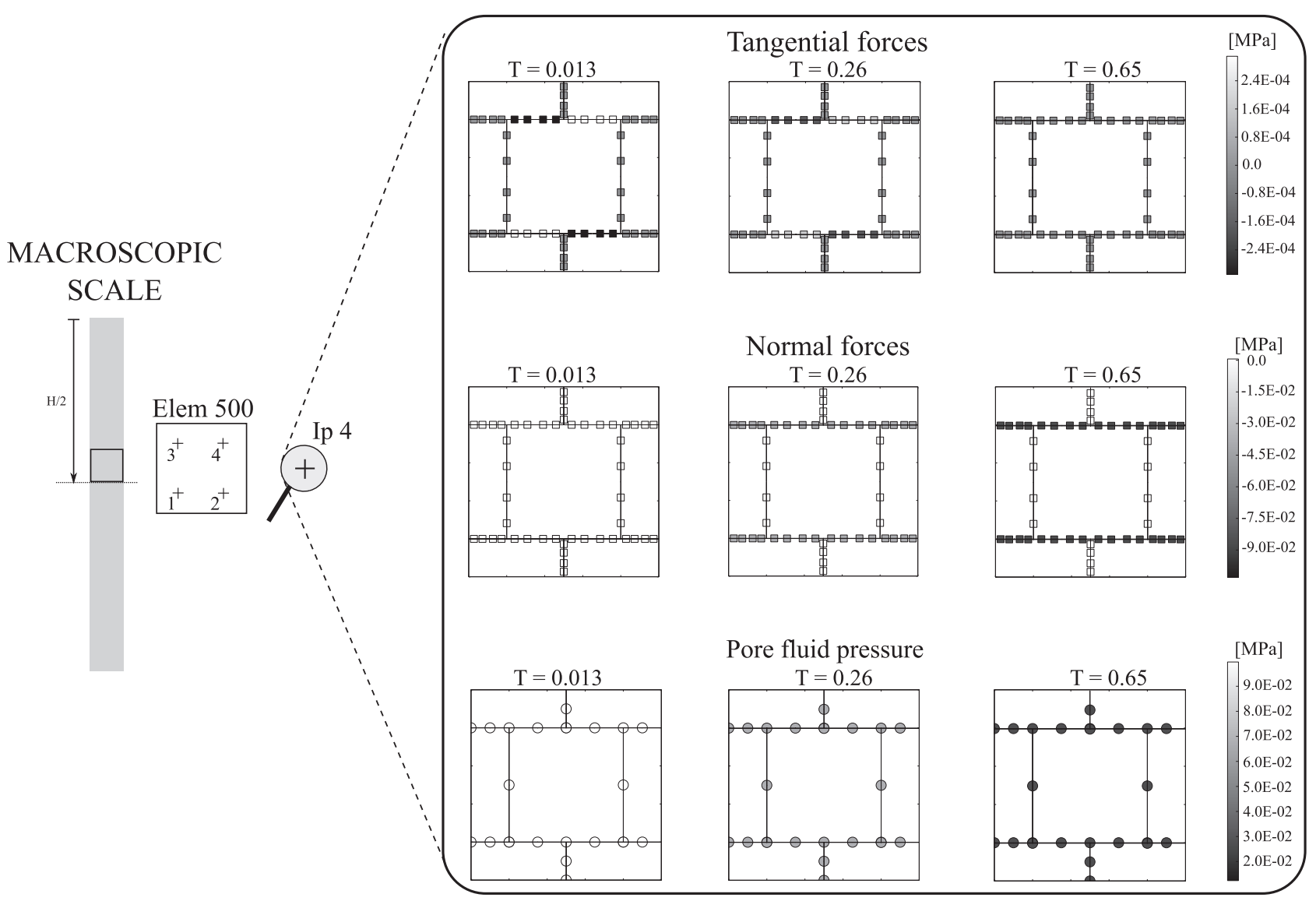

Fig. 15. Behavior of the microstructure with rectangular grains corresponding to the element 500 and the integration Gauss point 4 . 


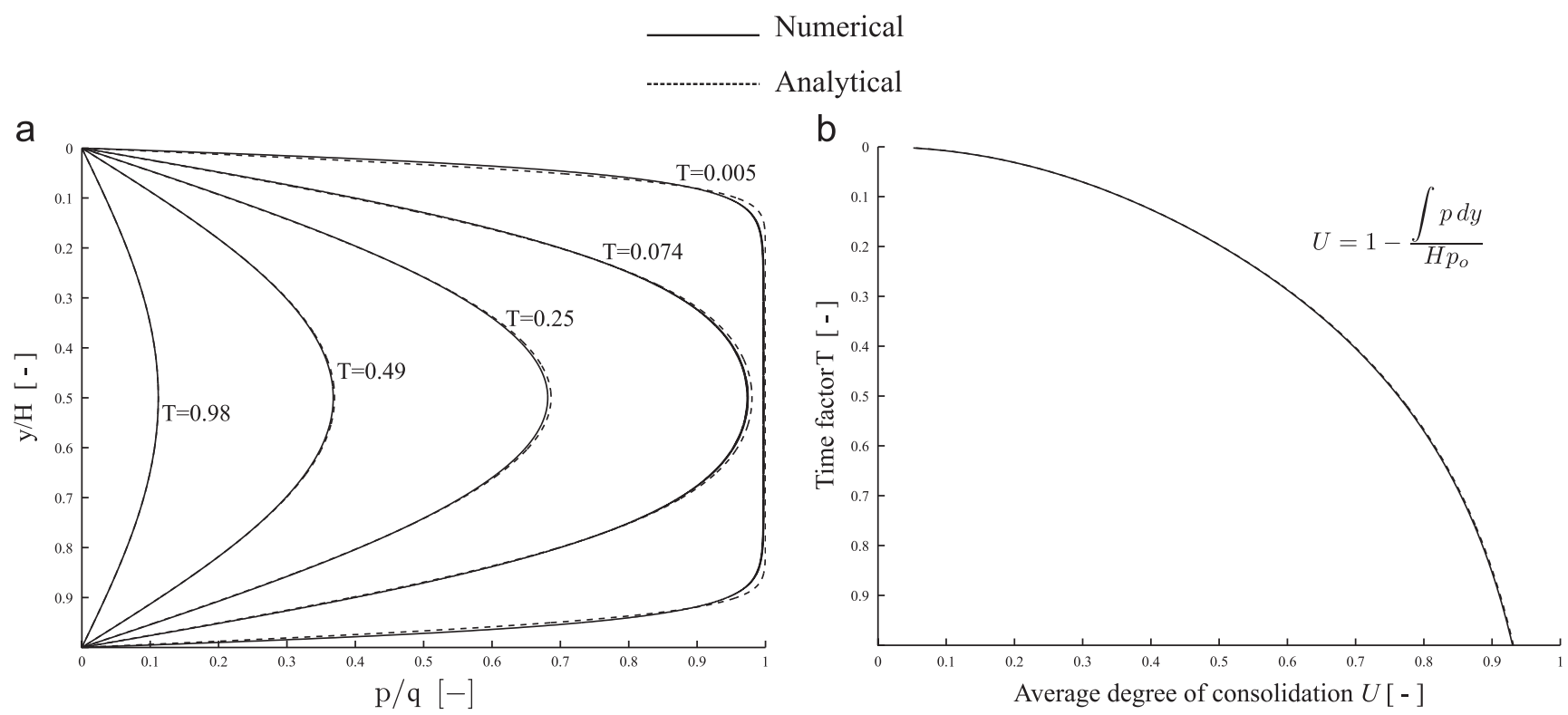

Fig. 16. Comparison between the numerical results and the consolidation Biot theory: (a) normalized fluid pressure and (b) average degree of consolidation U.
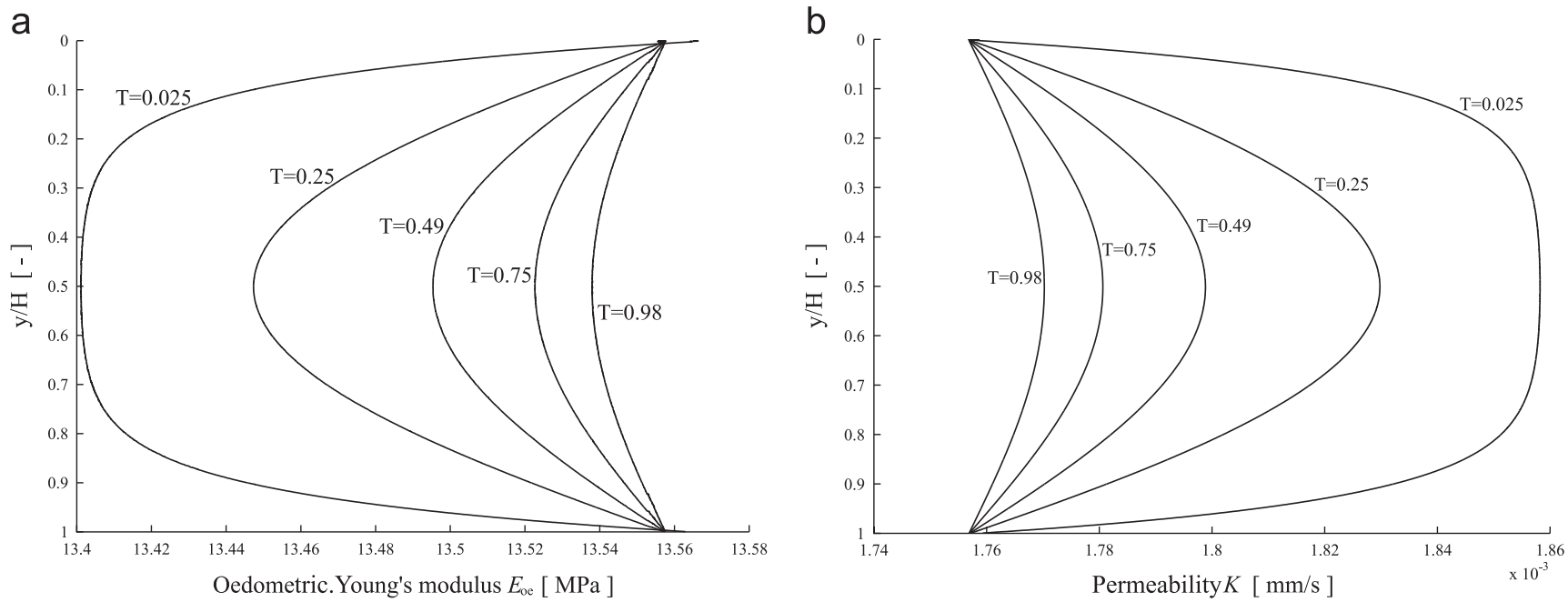

Fig. 17. Homogenized hydromechanical parameters with the microstructure composed by irregular grains: (a) Young modulus $E_{o e}$ and (b) permeability $K$.

[60]. In this manner, by considering a proper description of the microstructure, the $\mathrm{FE}^{2}$ approach allows to reproduce complex features such as anisotropy by taking advantage of avoiding complex analytical formulations.

The convergence rate for this case is quadratic as well, and the time step problem is solved with a constant value of the iteration number equal to three. As previously proposed for a microstructure with rectangular grains, the behavior of one microstructure with irregular grains is reported in Fig. 18.

In order to further investigate possible changes of the hydromechanical behavior due to more complex microstructures, an additional set of computations is performed with microstructures characterized by randomized shapes of the elastic grains (Fig. 19). For each microstructure the computations are repeated with different stiffness of the cohesive interfaces, the same employed in the numerical simulations presented in Fig. 13.

The hydromechanical properties are illustrated in Fig. 20 and show a similar behavior of the homogenized parameters presented for the microstructure with rectangular grains plotted in Fig. 12. The qualitative trend of the pore pressure dissipation is characterized by the same evolution plotted in Fig. 13 and, for this reason, the results are not included hereafter.

It is important to remark that, for the presented poroelastic problem, the complexity of the microstructure does not provide an important contribution to the non-classical coupling terms defined in Eq. (27) (i.e. the terms $B_{222}, K_{222}, L_{2}$ and $J_{2}$ ) which further emphasize the Biot-like characteristics of the computed equivalent porous medium. Nevertheless, in presence of more complex stress paths and damaged interfaces such coupling terms may appear in the numerical definition of the hydromechanical constitutive equations (e.g. the terms $K_{222}$ will not tend necessarily to zero as in this elastic problem).

\subsection{Computational cost}

The main limitation of the numerical homogenization method is represented by the computational cost in terms of time since the finite element problem is solved on two observation scales. The computational time is further increased because of the perturbation method which involves the integration of the microscopic problem seven more times, each of them corresponding to a 


\section{MICROSCOPIC SCALE}

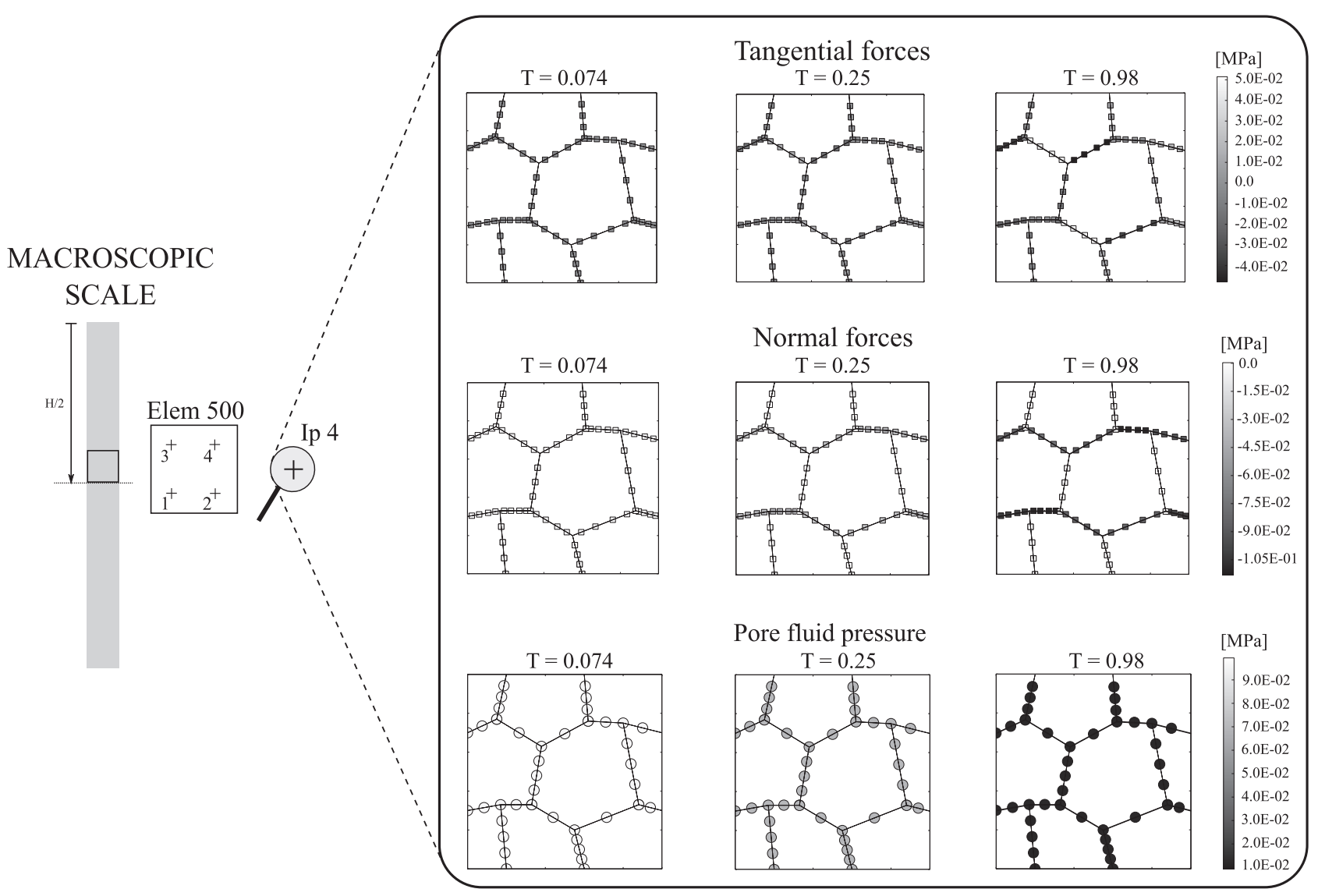

Fig. 18. Behavior of the microstructure with irregular grains corresponding to the element 500 and the integration Gauss point 4 .

perturbated component of the vector $\mathrm{d} U^{\tau 1}$ (Eq. (29)). For this particular initial boundary problem, the solution of a single time step is obtained in $7.5 \mathrm{~min}$ for the microstructure with rectangular grains and $47.0 \mathrm{~min}$ for the microstructure with irregular grains. In both cases the computations have been carried out with an Intel i7 $4770 \mathrm{~K}$ processor. For a given time step, all the computational time is spent to solve the microscopic finite element problem which has to be computed for all the Gauss points and for all iterations.

For elastic problems, a possible solution to decrease the computational time might be updating the stiffness matrix just at the first iteration for each time step. In this manner, the computational time employed to solve a time step decreases to $1.4 \mathrm{~min}$ and $8.0 \mathrm{~min}$ for the microstructure with rectangular and irregular grains, respectively. To optimize the computational time employed to derive the stiffness matrix, the static condensation method introduced in Kouznetsova et al. [55,24] for a single phase problem should be adopted for a coupled problem as proposed in van den Eijnden [56,61] to avoid the perturbation method.

\section{Conclusions}

In this work, a hydromechanical boundary value problem has been investigated by using a double scale model in which the macroscopic behavior of a finely periodic continuum is derived by means of a computational homogenization approach, the so-called finite element squared method $\mathrm{FE}^{2}$. At the microscopic observation scale the model formulated in Frey et al. [31] has been considered. In this approach the microstructure of the material is modeled as a

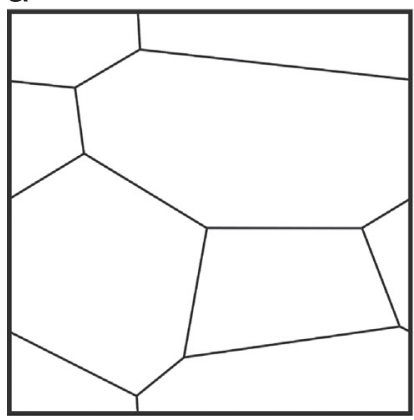

micro-A b

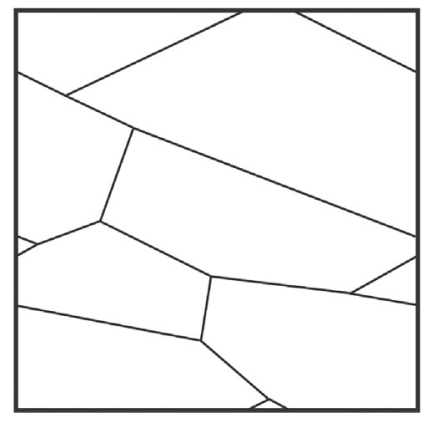

micro-B
$\mathrm{C}$

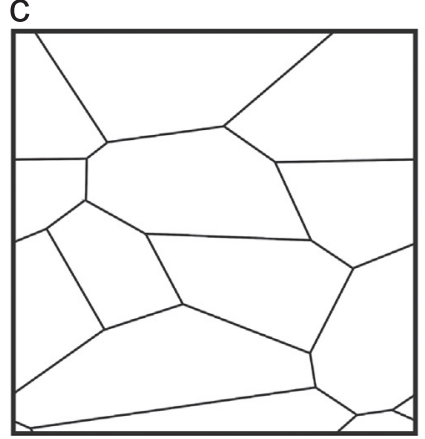

micro-C

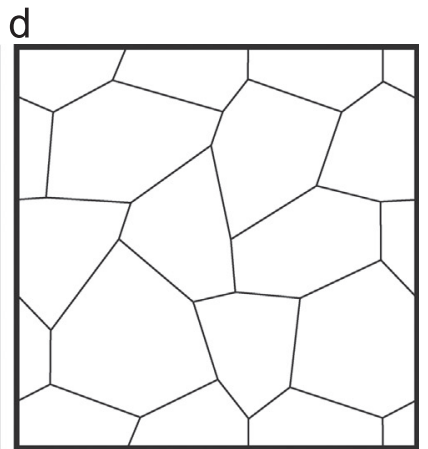

micro-D

Fig. 19. Microstructures characterized by a more randomized shape of the elastic grains. 


\section{Legend}
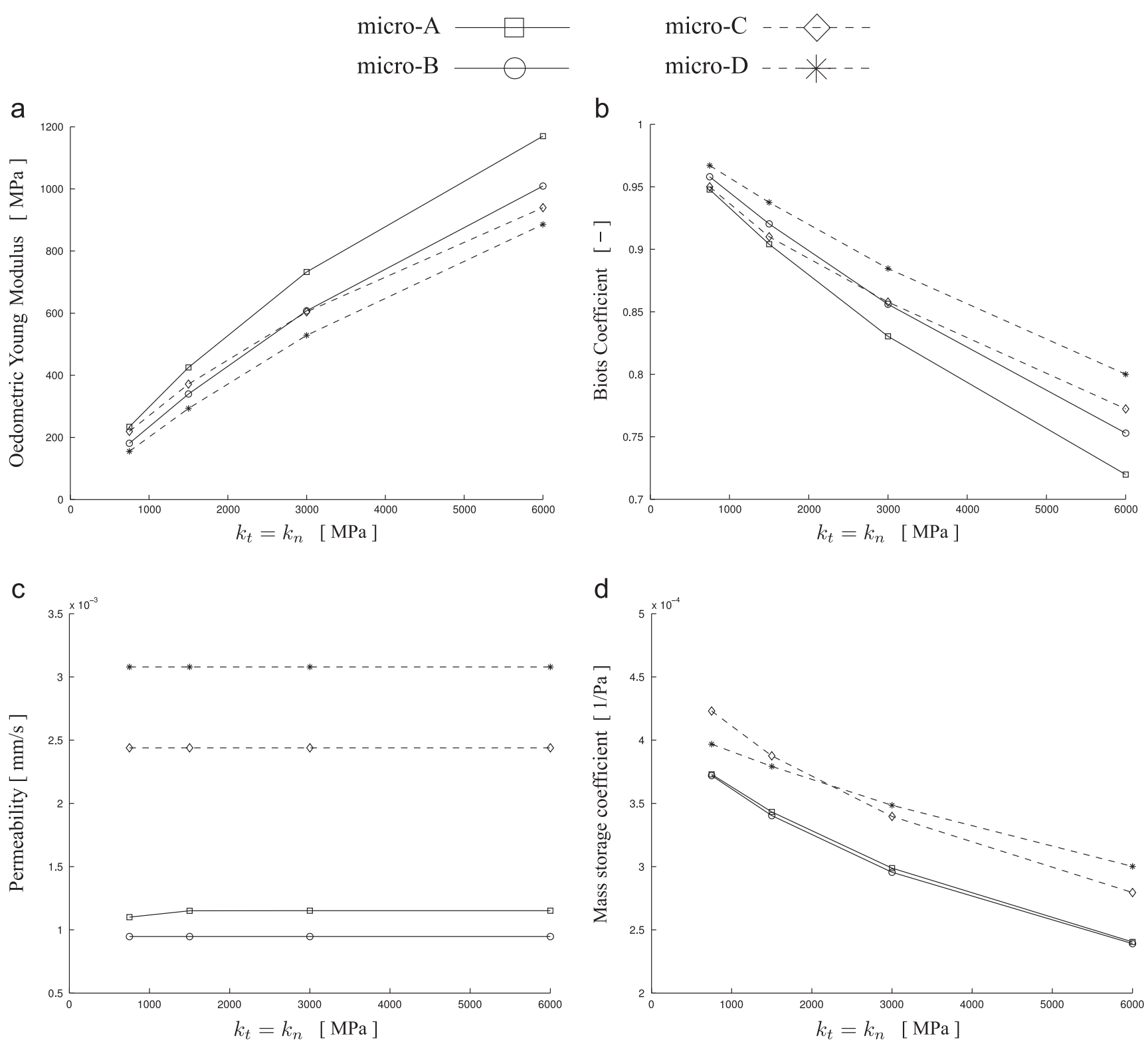

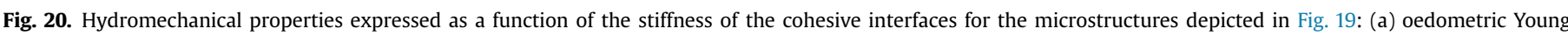
modulus, (b) Biot's coefficient, and (c) mass storage coefficient.

an assembly of hyperelastic grains and cohesive interfaces that identify a network of channels in which the fluid can percolate.

In the first part of this paper, the macroscopic formulation of a general hydromechanical finite element problem is presented to recall the main differences with the classical poromechanics theory in which, the constitutive hydromechanic behavior is defined with phenomenological assumptions on each component of the mixture and their reciprocal interaction. In the second part, a more extensive description of the microscopic model is provided in order to emphasize some differences with respect to the implementation proposed by Frey et al. [31].

To prove the consistency of the selected microscopic model and to validate the implementation in the finite element program Lagamine (code of the University of Liege), a one-dimensional consolidation problem is studied by considering two different microstructures. For this purposes, the numerical results are compared with the analytical solution for a Biot-like porous medium characterized by the homogenized hydromechanical properties obtained via numerical homogenization. As a result, it has been shown that the proposed multi-scale model is able to reproduce the hydromechanical behavior described by the classical Biot poromechanic theory [1].

The macroscopic constitutive formulation can take advantage of the microscopic material modeling in many aspects. Indeed, as presented in this paper, the $\mathrm{FE}^{2}$ method can overcome the phenomenological concept of effective stress and directly derive the behavior of the mixture by virtue of a proper microscopic description of the Representative Elementary Volume of a given material. By using this approach, it is possible to avoid complex phenomenological formulation used to describe coupled phenomena which are here a direct outcome of the homogenization process. Moreover, this method can be further extended to model the post/peak behavior of geomaterials by introducing a damage law in the interfaces behavior [57-59,48,31]. In this framework, 
a

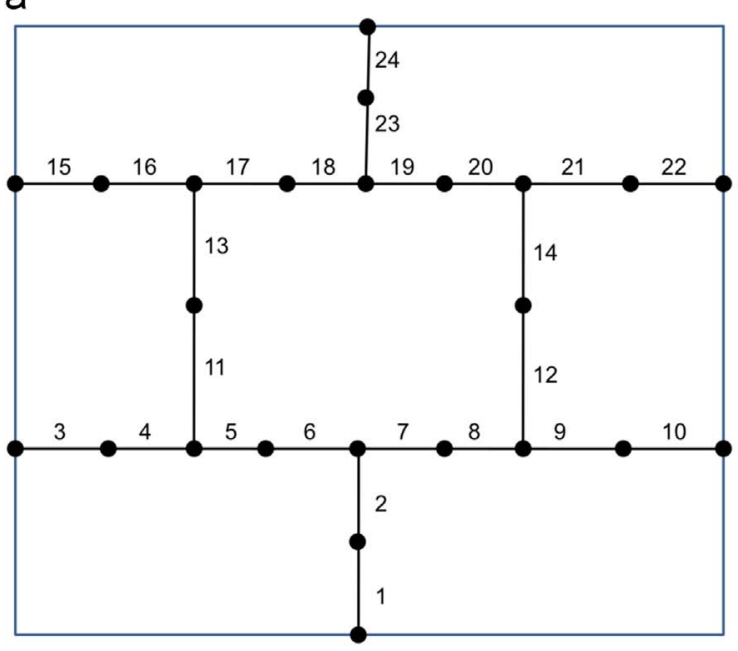

b

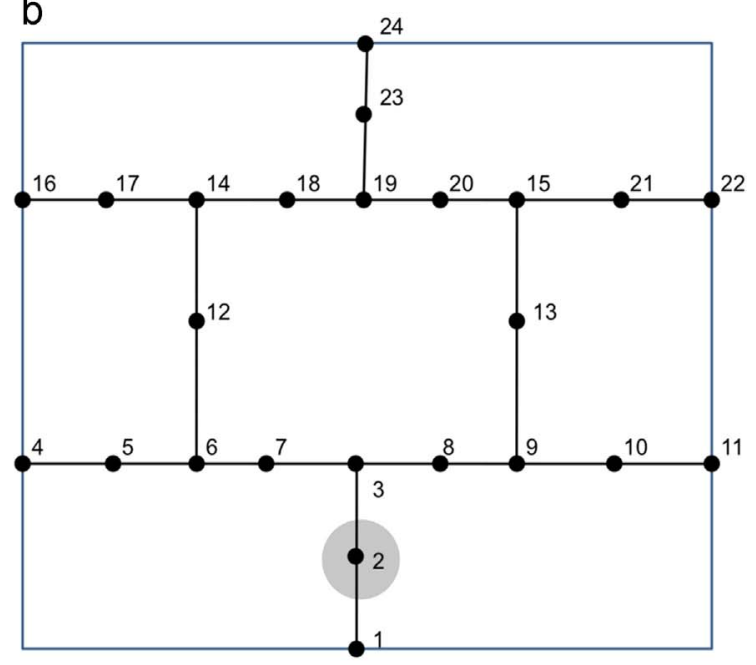

Fig. A1. Microstructure with rectangular grains: (a) numbering of the interfaces and (b) numbering of the fluid nodes.

the $\mathrm{FE}^{2}$ cannot escape to the pathological mesh dependency issue in strain localization modeling and high order continuum theories should be taken into account as proposed in [60]. As a result, an intrinsic dependency between the hydraulic permeability and the material degradation is generated.

For these reasons, by improving the constitutive behavior of each microstructural component and by considering more elaborated microstructures, the $\mathrm{FE}^{2}$ method can be considered as an advanced tool to formulate constitutive equations and to model multiphase coupling which characterizes complex geomaterial behavior.

\section{Appendix A}

An example of the entire set of equations composing the matrix $P_{i j}$ (Eq. (21)) to compute the pore pressure field for the microstructure composed by rectangular grains (Fig. A1) is given in this section. In the following equations the subscript indicates the number of a given node and the superscript indicates the corresponding number of the adjacent interface to that given node:

Node

$$
\begin{aligned}
& \text { 2. } \omega_{1}+\omega_{2}=0 \quad \phi^{1}\left(\lambda_{2}-\lambda_{1}\right)+\phi^{2}\left(\lambda_{2}-\lambda_{3}\right)=0 \\
& \text { 3. } \omega_{2}+\omega_{6}+\omega_{7}=0 \quad \phi^{2}\left(\lambda_{3}-\lambda_{2}\right)+\phi^{6}\left(\lambda_{3}-\lambda_{7}\right)+\phi^{7}\left(\lambda_{3}-\lambda_{8}\right)=0 \\
& \text { 5. } \omega_{3}+\omega_{4}=0 \quad \phi^{3}\left(\lambda_{5}-\lambda_{4}\right)+\phi^{4}\left(\lambda_{5}-\lambda_{6}\right)=0 \\
& \text { 6. } \omega_{4}+\omega_{5}+\omega_{11}=0 \quad \phi^{4}\left(\lambda_{6}-\lambda_{5}\right)+\phi^{5}\left(\lambda_{6}-\lambda_{7}\right)+\phi^{11}\left(\lambda_{6}-\lambda_{12}\right)=0 \\
& \text { 7. } \omega_{5}+\omega_{6}=0 \quad \phi^{5}\left(\lambda_{7}-\lambda_{6}\right)+\phi^{6}\left(\lambda_{7}-\lambda_{3}\right)=0 \\
& \text { 8. } \omega_{7}+\omega_{8}=0 \quad \phi^{7}\left(\lambda_{8}-\lambda_{3}\right)+\phi^{8}\left(\lambda_{8}-\lambda_{9}\right)=0 \\
& \text { 9. } \omega_{8}+\omega_{9}+\omega_{12}=0 \quad \phi^{8}\left(\lambda_{9}-\lambda_{8}\right)+\phi^{9}\left(\lambda_{9}-\lambda_{10}\right)+\phi^{12}\left(\lambda_{9}-\lambda_{13}\right)=0 \\
& \text { 10. } \omega_{9}+\omega_{10}=0 \quad \phi^{9}\left(\lambda_{10}-\lambda_{9}\right)+\phi^{10}\left(\lambda_{10}-\lambda_{11}\right)=0 \\
& \text { 12. } \omega_{11}+\omega_{13}=0 \quad \phi^{11}\left(\lambda_{12}-\lambda_{6}\right)+\phi^{13}\left(\lambda_{12}-\lambda_{14}\right)=0 \\
& \text { 13. } \omega_{12}+\omega_{14}=0 \quad \phi^{12}\left(\lambda_{13}-\lambda_{9}\right)+\phi^{14}\left(\lambda_{13}-\lambda_{15}\right)=0 \\
& \text { 14. } \omega_{13}+\omega_{16}+\omega_{17}=0 \quad \phi^{13}\left(\lambda_{14}-\lambda_{12}\right)+\phi^{16}\left(\lambda_{14}-\lambda_{17}\right) \\
& \quad+\phi^{17}\left(\lambda_{14}-\lambda_{18}\right)=0 \\
& \text { 15. } \omega_{14}+\omega_{20}+\omega_{21}=0 \quad \phi^{14}\left(\lambda_{15}-\lambda_{13}\right)+\phi^{20}\left(\lambda_{15}-\lambda_{20}\right) \\
& \quad+\phi^{21}\left(\lambda_{15}-\lambda_{21}\right)=0 \\
& \text { 17. } \omega_{15}+\omega_{16}=0 \quad \phi^{15}\left(\lambda_{17}-\lambda_{16}\right)+\phi^{16}\left(\lambda_{17}-\lambda_{14}\right)=0 \\
& \text { 18. } \omega_{17}+\omega_{18}=0 \quad \phi^{17}\left(\lambda_{18}-\lambda_{14}\right)+\phi^{18}\left(\lambda_{18}-\lambda_{19}\right)=0 \\
& \text { 19. } \omega_{18}+\omega_{19}+\omega_{23}=0 \quad \phi^{18}\left(\lambda_{19}-\lambda_{18}\right)+\phi^{19}\left(\lambda_{19}-\lambda_{20}\right) \\
& \quad+\phi^{23}\left(\lambda_{19}-\lambda_{23}\right)=0 \\
& \text { 20. } \omega_{19}+\omega_{20}=0 \quad \phi^{19}\left(\lambda_{20}-\lambda_{19}\right)+\phi^{20}\left(\lambda_{20}-\lambda_{15}\right)=0
\end{aligned}
$$

$$
\begin{array}{lrl}
\text { 21. } \omega_{21}+\omega_{22}=0 & \phi^{21}\left(\lambda_{21}-\lambda_{15}\right)+\phi^{22}\left(\lambda_{21}-\lambda_{22}\right)=0 \\
\text { 23. } \omega_{23}+\omega_{24}=0 & \phi^{23}\left(\lambda_{23}-\lambda_{19}\right)+\phi^{24}\left(\lambda_{23}-\lambda_{24}\right)=0
\end{array}
$$

Antiperiodic boundary conditions on flux mass:

1. $\omega_{1}+\omega_{24}=0 \quad \phi^{1}\left(\lambda_{1}-\lambda_{2}\right)+\phi^{24}\left(\lambda_{24}-\lambda_{23}\right)=0$

4. $\omega_{3}+\omega_{10}=0 \quad \phi^{3}\left(\lambda_{4}-\lambda_{5}\right)+\phi^{10}\left(\lambda_{11}-\lambda_{10}\right)=0$

16. $\omega_{15}+\omega_{22}=0 \quad \phi^{15}\left(\lambda_{16}-\lambda_{17}\right)+\phi^{22}\left(\lambda_{22}-\lambda_{21}\right)=0$

Periodic boundary conditions on the pore pressure field $\left(p_{j}-p_{j-1}=\Delta p^{\text {macro }}\right)$ :

11. $\lambda_{11}-\lambda_{4}=\Delta p^{\text {macro }, 2}=\frac{\partial p}{\partial x_{2}} \bar{a}$
22. $\lambda_{22}-\lambda_{16}=\Delta p^{\text {macro }, 2}=\frac{\partial p}{\partial x_{2}} \bar{a}$
24. $\lambda_{24}-\lambda_{1}=\Delta p^{\text {macro }, 1}=\frac{\partial p}{\partial x_{1}} \bar{a}$

\section{References}

[1] M. Biot, General theory of three-dimensional consolidation, J. Appl. Phys. 12 (1941) 155-164.

[2] G. Viggiani, S. Hall, E. Romero, Advanced experimental techniques in geomechanics, ALERT Doctoral School 2012, 2012.

[3] Y. Song, C. Davy, D. Troadec, A.-M. Blanchenet, F. Skoczylas, J. Talandier, J. Robinet, Multi-scale pore structure of $\{\mathrm{COx}\}$ claystone: towards the prediction of fluid transport, Mar. Pet. Geol. 65 (2015) 63-82.

[4] J.D. Eshelby, The determination of the elastic field of an ellipsoidal inclusion, and related problems, Proc. R. Soc. Lond. Ser. A Math. Phys. Sci. 241 (1957) 376-396.

[5] T. Mori, K. Tanaka, Average stress in matrix and average elastic energy of materials with misfitting inclusions, Acta Metall. 21 (1973) 571-574.

[6] M. Hori, S. Nemat-Nasser, Double-inclusion model and overall moduli of multi-phase composites, Mech. Mater. 14 (1993) 189-206.

[7] A.V. Hershey, The elasticity of an isotropic aggregate of anisotropic cubic crystals, J. Appl. Mech. 21 (3) (1954) 236.

[8] R. Hill, A self-consistent mechanics of composite materials, J. Mech. Phys. Solids 13 (1965) 213-222.

[9] Z. Hashin, The elastic moduli of heterogeneous materials, J. Appl. Mech. 29 (1) (1962) 143-150.

[10] R. Christensen, K. Lo, Solutions for effective shear properties in three phase sphere and cylinder models, J. Mech. Phys. Solids 27 (1979) 315-330.

[11] Z. Hashin, Analysis of composite materials, a survey, ASME. J. Appl. Mech. 50 (3) (1983) 481-505.

[12] J.L. Auriault, E. Sanchez-Palencia, Etude du comportement macroscopique d'un milieu poreux saturé déformable, J. Méc. 16 (1977) 576-603.

[13] A. Bensoussan, J.L. Lions, G. Papanicolaou, Asymptotic Analysis for Periodic Structures, North Holland, Amsterdam, 1978.

[14] E. Sanchez-Palencia, Non-Homogeneous Media and Vibration Theory, Fluid Flow in Porous Media, Springer-Verlag, Berlin, 1980. 
[15] S. Nemat-Nasser, M. Hori, Micromechanics: overall properties of heterogeneous materials, in: North-Holland Series in Applied Mathematics and Mechanics, vol. 37, Amsterdam, 1993.

[16] V. Deudé, L. Dormieux, D. Kondo, S. Maghous, Micromechanical approach to nonlinear poroelasticity: application to cracked rocks, J. Eng. Mech. 128 (2002) $848-855$.

[17] K. Terada, N. Kikuchi, Non linear application method for practical application, in: S. Ghosh, M. Ostoja-Starzewski (Eds.), ASME International Mechanical Engineering Congress and Exposition, 1995, p. 185.

[18] C. Miehe, J. Schroder, J. Schotte, Computational homogenization analysis in finite plasticity simulation of texture development in polycrystalline materials, Comput. Methods Appl. Mech. Eng. 171 (1999) 387-418.

[19] C. Miehe, J. Schotte, J. Schröder, Computational micro-macro transitions and overall moduli in the analysis of polycrystals at large strains, Comput. Mater. Sci. 16 (1999) 372-382.

[20] R. Smit, W. Brekelmans, H. Meijer, Prediction of the mechanical behavior of nonlinear heterogeneous systems by multi-level finite element modeling, Comput. Methods Appl. Mech. Eng. 155 (1998) 181-192.

[21] F. Feyel, Multiscale \{FE2\} elastoviscoplastic analysis of composite structures, Comput. Mater. Sci. 16 (1999) 344-354.

[22] F. Feyel, J.-L. Chaboche, Fe2 multiscale approach for modelling the elastoviscoplastic behaviour of long fibre sic/ti composite materials, Comput. Methods Appl. Mech. Eng. 183 (2000) 309-330.

[23] V. Kouznetsova, M.G.D. Geers, W.A.M. Brekelmans, Multi-scale constitutive modelling of heterogeneous materials with a gradient-enhanced computational homogenization scheme, Int. J. Numer. Methods Eng. 54 (2002) $1235-1260$.

[24] V.G. Kouznetsova, M.G.D. Geers, W.A.M. Brekelmans, Multi-scale second-order computational homogenization of multi-phase materials: a nested finite element solution strategy, Comput. Methods Appl. Mech. Eng. 193 (2004) $5525-5550$.

[25] M. Geers, V. Kouznetsova, W. Brekelmans, Multi-scale computational homogenization: trends and challenges, J. Comput. Appl. Math. 234 (2010) 2175-2182, Fourth International Conference on Advanced Computational Methods in Engineering (ACOMEN 2008).

[26] D.J. Luscher, D.L. McDowell, C.A. Bronkhorst, A second gradient theoretical framework for hierarchical multiscale modeling of materials, Int. J. Plast. 26 (2010) 1248-1275, Special Issue In Honor of Lallit Anand.

[27] J. Schröder, A numerical two-scale homogenization scheme: the fe2-method, in: J. Schröder, K. Hackl (Eds.), Plasticity and Beyond, CISM International Centre for Mechanical Sciences, vol. 550, Springer, Vienna, 2014, pp. 1-64.

[28] I. Özdemir, W.A.M. Brekelmans, M.G.D. Geers, Computational homogenization for heat conduction in heterogeneous solids, Int. J. Numer. Methods Eng. 73 (2008) 185-204.

[29] F. Su, F. Larsson, K. Runesson, Computational homogenization of coupled consolidation problems in micro-heterogeneous porous media, Int. J. Numer. Methods Eng. 88 (2011) 1198-1218.

[30] T.J. Massart, A.P.S. Selvadurai, Stress-induced permeability evolution in a quasi-brittle geomaterial, J. Geophys. Res.: Solid Earth 117 (2012) B07207.

[31] J. Frey, R. Chambon, C. Dascalu, A two-scale poromechanical model for cohesive rocks, Acta Geotech. 8 (2013) 107-124.

[32] R. Charlier, Approche unifiée de quelques problemes non lineaires de mécanique des milieux conutinus par la méthode des éléments finis (Ph.D. thesis), Université de Liège, 1986-1987.

[33] F. Collin, Couplages thermo-hydro-mécaniques dans les sols et les roches tendres partiellement saturés (Ph.D. thesis), Université de Liège, Faculté de Science Apppliquées, 2003

[34] E. Catalano, B. Chareyre, E. Barthelemy, Pore-scale modeling of fluid-particles interaction and emerging poromechanical effects, Int. J. Numer. Anal. Methods Geomech. 38 (2014) 51-71.

[35] O.C. Zienckiewicz, R. Taylor, The Finite Element Method Fifth edition Volume 1: The Basis, McGraw-Hill, Butterworth-Heinemann: Stonchem, MA, 2000.

[36] O. Coussy, Poromechanics, Laboratoire Central des Ponts et Chausées, Paris, 2004.
[37] R.W. Lewis, B.A. Schrefler, The Finite Element Method in the Static and Dynamic Deformation and Consolidation of Porous Media, Wiley, New York, 1999.

[38] J.L. Auriault, C. Geindreau, P. Royer, J. Bloch, Poromechanics II, CRC Press, Grenoble, 2002.

[39] R. de Boer, Theory of Porous Media, Springer, Berlin, 2000.

[40] R. de Boer, Theory of Porous Media: Highlights in Historical Development and Current State, Springer Science \& Business Media, Berlin, 2012.

[41] F. Collin, R. Chambon, R. Charlier, A finite element method for poro mechanica modelling of geotechnical problems using local second gradient models, Int. J. Numer. Methods Eng. 65 (2006) 1749-1772.

[42] R.I. Borja, E. Alarcón, A mathematical framework for finite strain elastoplastic consolidation part 1: balance laws, variational formulation, and linearization, Comput. Methods Appl. Mech. Eng. 122 (1995) 145-171.

[43] R.I. Borja, C. Tamagnini, E. Alarcón, Elastoplastic consolidation at finite strain part 2: finite element implementation and numerical examples, Comput. Methods Appl. Mech. Eng. 159 (1998) 103-122.

[44] T. Matsushima, R. Chambon, D. Caillerie, Large strain finite element analysis of a local second gradient: application, Int. J. Numer. Methods Eng. 54 (2002) 499-521.

[45] Y. Sieffert, O. Buzzi, F. Collin, Numerical study of shear band instability and effect of cavitation on the response of a specimen under undrained biaxial loading, Int. J. Solids Struct. 51 (2014) 1686-1696.

[46] K. Terada, M. Hori, T. Kyoya, N. Kikuchi, Simulation of the multi-scale convergence in computational homogenization approaches, Int. J. Solids Struct. 37 (2000) 2285-2311.

[47] O. van der Sluis, P. Schreurs, W. Brekelmans, H. Meijer, Overall behaviour of heterogeneous elastoviscoplastic materials: effect of microstructural modelling, Mech. Mater. 32 (2000) 449-462.

[48] G. Bilbie, C. Dascalu, R. Chambon, D. Caillerie, Micro-fracture instabilities in granular solids, Acta Geotech. 3 (2008) 25-35.

[49] J. Bonet, R.D. Wood, Nonlinear Continuum Mechanics for Finite Elemen Analysis, Cambridge University Press, Cambridge, 1997.

[50] V. Reichenberger, H. Jakobs, P. Bastian, R. Helmig, A mixed-dimensional finite volume method for two-phase flow in fractured porous media, Adv. Water Res. 29 (2006) 1020-1036.

[51] Q. Kang, D. Zhang, S. Chen, Unified lattice Boltzmann method for flow in multiscale porous media, Phys. Rev. E 66 (5) (2002).

[52] Y. Han, P.A. Cundall, Lattice Boltzmann modeling of pore-scale fluid flow through idealized porous media, Int. J. Numer. Methods Fluids 67 (2011) 1720-1734.

[53] C. Felippa, K. Park, Staggered transient analysis procedures for coupled mechanical systems: formulation, Comput. Methods Appl. Mech. Eng. 24 (1980) 61-111.

[54] K.C. Park, C.A. Felippa, Partitioned transient analysis procedures for coupledfield problems: accuracy analysis, J. Appl. Mech. December 1 (1980) 47 (4) 919

[55] V. Kouznetsova, W.A.M. Brekelmans, F.P.T Baaijens, An approach to micromacro modeling of heterogeneous materials, Comput. Mech. 27 (2001) 37-48.

[56] B. van den Eijnden, Multi-scale modelling of the hydromechanical behaviour of argillaceous rocks (Ph.D. thesis), Université de Grenoble, 2015.

[57] A. Needleman, A continuum model for void nucleation by inclusion debonding, J. Appl. Mech. 54 (1987) 525-531.

[58] V. Tvergaard, Effect of fibre debonding in a whisker-reinforced metal, Mater Sci. Eng.: A 125 (1990) 203-213.

[59] V. Tvergaard, Cohesive zone representations of failure between elastic or rigid solids and ductile solids, Eng. Fract. Mech. 70 (2003) 1859-1868.

[60] A.P. van den Eijnden, P. Bésuelle, F. Collin, R. Chambon, J. Desrues, Modeling the strain localization around an underground gallery with a hydromechanical double scale model: effect of anisotropy. 2016 (submitted).

[61] A.P. van den Eijnden, P. Bésuelle, F. Collin, R. Chambon, A FE² modelling approach to hydromechanical coupling in cracking-induced localization problems for granular solids. Int. J. Solids Struct. 2016 (submitted). 